\title{
Discrete semiclassical orthogonal polynomials of class 2
}

\author{
Diego Dominici * \\ Johannes Kepler University Linz \\ Doktoratskolleg "Computational Mathematics" \\ Altenberger Straße 69, 4040 Linz, Austria. \\ Permanent address: Department of Mathematics \\ State University of New York at New Paltz \\ 1 Hawk Dr., New Paltz, NY 12561-2443, USA \\ Francisco Marcellán ${ }^{\dagger}$ \\ Departamento de Matemáticas \\ Universidad Carlos III de Madrid \\ Escuela Politécnica Superior \\ Av. Universidad 30 \\ 28911 Leganés \\ Spain
}

April 29, 2019

\begin{abstract}
In this contribution, discrete semiclassical orthogonal polynomials of class $s \leq 2$ are studied. By considering all possible solutions of the Pearson equation, we obtain the canonical families in each class. We also consider limit relations between these and other families of orthogonal polynomials.
\end{abstract}

${ }^{*}$ e-mail: diego.dominici@dk-compmath.jku.at

†e-mail: pacomarc@ing.uc3m.es 


\section{Introduction}

Semiclassical linear functionals with respect to the derivative operator have received increasing attention in the literature of orthogonal polynomials taking into account their connections with many interesting problems in mathematical physics and numerical quadratures. From the so called Pearson equation associated with those linear functionals, ladder operators for the corresponding sequences of orthogonal polynomials can be deduced from the structure relations they satisfy (see [36], [37], [39]). As a direct consequence, a simple computation allows to deduce the corresponding holonomic equations, i.e. second order linear differential equations with polynomial coefficients of bounded degrees (depending on the degree of the polynomial) associated with the class of the linear functional (see [30], [39]).

The case of semiclassical linear functionals with respect to the difference operator in a uniform lattice is related to discretizations of holonomic equations, a fact pointed out in the classical case by Nikiforov, Uvarov and Suslov in a stimulating monograph on classical discrete orthogonal polynomials [42].

In both situations, a key problem is the classification of semiclassical linear functionals by using a hierarchy according to their class. This represents an alternative method to the Askey tableau, where classical orthogonal polynomials appear as a hierarchy of hypergeometric polynomials (see [31]).

Examples of semiclassical linear functionals with respect to the derivative operator when the class is either $s=1$ (see [9]) or $s=2$ (see [35]) have been studied in the literature. Nevertheless, some of them are related to perturbations of classical linear functionals by the addition of Dirac functionals, or their derivatives, supported on convenient points ([2], [33]). The important fact is that these orthogonal polynomials, the so called Krall-type orthogonal polynomials, are eigenfunctions of higher order differential operators. They are related to bispectral problems and have been intensively used in the generation of exceptional polynomials, an useful tool in the study of integrable systems. 
In the discrete case, there is a limited development in these topics (see [34]). The case $s=0$ has been deeply studied by many authors (see [1], 24] ) and the approach to the case $s=1$ has been done by Dominici and Marcellán in a recent paper (see [19]). Some examples of semiclassical discrete polynomials for $s=1$ appeared in [47].

The aim of this contribution is twofold. First, we deal with three different perturbations of discrete linear functionals based on the so called linear spectral transformations (Uvarov, Christoffel and Geronimus) [50], truncations and symmetrization processes, respectively. Second, from the above transformations we are able to generate semiclassical linear functionals of class $s=1$ and $s=2$. Some of them appear in a natural way in the literature but others are new. We put attention only in the representation of the corresponding Stieltjes functions and, as a consequence, we deduce the corresponding class. Notice that an interesting and open problem is to analyze the coefficients of the three term recurrence relation they satisfy, in particular the so called Laguerre-Freud equations. For generalized Charlier polynomials such equations appeared in the monograph by W. Van Assche [49] as well as in [29]. They lead to limiting cases of the discrete Painlevé equation corresponding to $D_{4}^{c}$ in the Sakai's classification. A generalization of the Krawtchouk polynomials and the fifth Painlevé equation are studied in [10]. Some interesting examples of semiclassical extensions of Meixner polynomials appeared in [11] and [22]. They yield a limit case of asymmetric discrete Painlevé IV equations. Discrete orthogonal polynomials with respect to the hypergeometric weight have been analyzed in [21] and [17].

The structure of the paper is the following. In Section 2, a basic background about discrete semiclassical linear functionals is given. In particular, the class is defined from the degrees of polynomials appearing in the discrete Pearson equation they satisfy. We emphasize the role of the Stieltjes function associated with every linear functional. In the semiclassical case, the corresponding Stieltjes function satisfies a first order linear difference equation with polynomial coefficients. They provide the key information about the class of the linear functional. Section 3 deals with the three canonical perturbations of linear functionals we will deal in the sequel. The corresponding Stieltjes functions are deduced. In Section 4, classical discrete linear functionals are revisited. In Section 5 we analyze semiclassical linear functionals of 
class $s=1$ in such a way that the results given in [19] are completed. Finally, in Section 6, we study examples of discrete semiclassical linear functionals of class $s=2$ generated by the three canonical perturbations introduced in Section 3 .

\section{Basic background on discrete semiclassical linear functionals}

Let $\mathbb{N}_{0}$ denote the set $\mathbb{N} \cup\{0\}=0,1,2, \ldots$. Throughout the paper we will use the notation

$$
\begin{gathered}
\mathbf{a}=\left(a_{1}, \ldots, a_{p}\right), \quad \mathbf{b}=\left(b_{1}, \ldots, b_{q}\right), \quad p, q \in \mathbb{N}_{0}, \\
x+\mathbf{a}=\prod_{i=1}^{p}\left(x+a_{i}\right), \quad x+\mathbf{b}=\prod_{i=1}^{q}\left(x+b_{i}\right),
\end{gathered}
$$

and

$$
(\mathbf{a})_{n}=\prod_{i=1}^{p}\left(a_{i}\right)_{n}, \quad(\mathbf{b})_{n}=\prod_{i=1}^{q}\left(b_{i}\right)_{n}, \quad n \in \mathbb{N}_{0},
$$

where the Pochhammer polynomial $(x)_{n}$ is defined by $(x)_{0}=1$ and [43, 18:12]

$$
(x)_{n}=\prod_{j=0}^{n-1}(x+j), \quad n \in \mathbb{N} .
$$

From (2), we immediately obtain the recurrence

$$
(x)_{n+1}=(x+n)(x)_{n}, \quad n \in \mathbb{N}_{0} .
$$

On the other hand, if we consider the analytic continuation with $\alpha \in \mathbb{C}$, then

$$
(x)_{\alpha}=\frac{\Gamma(x+\alpha)}{\Gamma(x)}, \quad-(x+\alpha) \notin \mathbb{N}_{0},
$$

where $\Gamma(x)$ denotes the Gamma function [44, Chapter 5.].

In this work, we deal with linear functionals defined in $\mathbb{C}[x]$, the linear space of polynomials with complex coefficients. Indeed,

$$
L[r]=\sum_{x=0}^{\infty} r(x) \varrho(x), \quad r \in \mathbb{C}[x],
$$


where the weight function $\varrho: \mathbb{N}_{0} \rightarrow \mathbb{C}$ is

$$
\varrho(x)=\frac{(\mathbf{a})_{x}}{(\mathbf{b}+1)_{x}} \frac{z^{x}}{x !} .
$$

Using (3) , we see that the weight function $\varrho(x)$ satisfies the Pearson equation

$$
\frac{\varrho(x+1)}{\varrho(x)}=\frac{\eta(x)}{\sigma(x+1)},
$$

where the polynomials $\eta(x), \sigma(x)$ are

$$
\eta(x)=z(x+\mathbf{a}), \quad \sigma(x)=x(x+\mathbf{b}) .
$$

Note that from (1) we have $\operatorname{deg}(\eta)=p$ and $\operatorname{deg}(\sigma)=q+1$ and we assume they are coprime.

The linear functional $L$ (and also $\varrho$ ) is said to be semiclassical. The class of $L$ (and also $\varrho$ ) is the non-negative integer number

$$
s=\max \{\operatorname{deg}(\sigma)-2, \operatorname{deg}(\sigma-\eta)-1\} .
$$

The linear functionals of class $s=0$ are called discrete classical 24 linear functionals.

The number $s$ depends on $p, q$, and $z$. It follows from (8) that we have four cases to consider:

1. $p>q+1$. In this case, $\operatorname{deg}(\sigma-\eta)-1=p-1>q$ and $s=$ $\max \{q-1, p-1\}=p-1$.

2. $p<q+1$. In this case, $\operatorname{deg}(\sigma-\eta)-1=q$ and $s=\max \{q-1, q\}=q$.

3. $q+1=p, z \neq 1$. In this case, $\operatorname{deg}(\sigma-\eta)-1=q$ and $s=\max \{q-1, q\}=$ $q$.

4. $q+1=p, z=1$. In this case, $\operatorname{deg}(\sigma-\eta)-1=q-1$ and $s=$ $\max \{q-1, q-1\}=q-1$.

Thus, we conclude that

$$
s= \begin{cases}p-1, & p>q+1 \\ q, & p<q+1 \\ q, & p=q+1, \quad z \neq 1 \\ q-1, & p=q+1, \quad z=1\end{cases}
$$


Note that we can also write $s$ as

$$
s= \begin{cases}\max \{\operatorname{deg}(\sigma)-1, \operatorname{deg}(\sigma-\eta)-1\}, & \operatorname{deg}(\sigma-\eta) \neq \operatorname{deg}(\sigma)-1 \\ \operatorname{deg}(\sigma)-2, & \operatorname{deg}(\sigma-\eta)=\operatorname{deg}(\sigma)-1\end{cases}
$$

The Stieltjes transform of the linear functional $L$ is defined by

$$
S(t)=L\left[\frac{1}{t-x}\right]
$$

Here the linear functional is acting on $x$. The connection between $\eta(x), \sigma(x)$ and $S(t)$ is given by the following result.

Lemma 1 The Stieltjes transform $S(t)$ of the linear functional $L$ satisfies the difference equation

$$
\sigma(t+1) S(t+1)-\eta(t) S(t)=\xi(t)
$$

where $\xi(t)$ is a polynomial of degree s that is the class of the linear functional.

Proof. From (11), we have

$$
\begin{gathered}
\sigma(t+1) S(t+1)-\eta(t) S(t)=\sigma(t+1) \sum_{x=0}^{\infty} \frac{\varrho(x)}{t+1-x}-\eta(t) \sum_{x=0}^{\infty} \frac{\varrho(x)}{t-x} \\
=\frac{\sigma(t+1)}{t+1}+\sigma(t+1) \sum_{x=1}^{\infty} \frac{\varrho(x)}{t+1-x}-\eta(t) \sum_{x=0}^{\infty} \frac{\varrho(x)}{t-x} \\
=\frac{\sigma(t+1)}{t+1}+\sigma(t+1) \sum_{x=0}^{\infty} \frac{\varrho(x+1)}{t-x}-\eta(t) \sum_{x=0}^{\infty} \frac{\varrho(x)}{t-x} .
\end{gathered}
$$

Using the Pearson equation (7), we get

$$
\begin{gathered}
\sigma(t+1) S(t+1)-\eta(t) S(t)=\frac{\sigma(t+1)}{t+1}+\sigma(t+1) \sum_{x=0}^{\infty} \frac{\eta(x)}{\sigma(x+1)} \frac{\varrho(x)}{t-x}-\eta(t) \sum_{x=0}^{\infty} \frac{\varrho(x)}{t-x} \\
=\frac{\sigma(t+1)}{t+1}+\sum_{x=0}^{\infty} \frac{\sigma(t+1) \eta(x)-\eta(t) \sigma(x+1)}{\sigma(x+1)} \frac{\varrho(x)}{t-x} .
\end{gathered}
$$

But

$$
\lambda_{x}(t)=\frac{\sigma(t+1) \eta(x)-\eta(t) \sigma(x+1)}{t-x}
$$


is a polynomial in $t$ (with coefficients depending on $x$ ) of degree deg $(\sigma-\eta)-$ 1. Since $\eta(x) \neq \sigma(x+1)$ (otherwise $\varrho$ is a constant function), we have $\operatorname{deg}(\sigma-\eta) \neq \operatorname{deg}(\sigma)-1$.

Therefore,

$$
\xi(t)=\frac{\sigma(t+1)}{t+1}+\sum_{x=0}^{\infty} \lambda_{x}(t) \frac{\varrho(x)}{\sigma(x+1)}
$$

is a polynomial in $t$ of degree $\max \{\operatorname{deg}(\sigma)-1, \operatorname{deg}(\sigma-\eta)-1\}$. From (10) we conclude that $\operatorname{deg}(\xi)=s$.

Let the falling factorial polynomials be defined by $\phi_{0}(x)=1$ and

$$
\phi_{n}(x)=\prod_{j=0}^{n-1}(x-j), \quad n \in \mathbb{N} .
$$

Comparing (14) with (21), we see that

$$
\phi_{n}(x)=(-1)^{n}(-x)_{n}=(x-n+1)_{n}, n \in \mathbb{N}_{0},
$$

or, using (44),

$$
\phi_{n}(x)=\frac{\Gamma(x+1)}{\Gamma(x-n+1)}=\frac{x !}{(x-n) !}=n !\left(\begin{array}{l}
x \\
n
\end{array}\right), \quad n \in \mathbb{N}_{0} .
$$

We define the moments of $L$ with respect to the basis $\left\{\phi_{n}(x)\right\}_{n \geq 0}$ by

$$
\nu_{n}=L\left[\phi_{n}\right], \quad n \in \mathbb{N}_{0} .
$$

From (6) and (16), we have

$$
\nu_{n}=\sum_{x=n}^{\infty} \frac{(\mathbf{a})_{x}}{(\mathbf{b}+1)_{x}} \frac{z^{x}}{(x-n) !}=\sum_{x=0}^{\infty} \frac{(\mathbf{a})_{x+n}}{(\mathbf{b}+1)_{x+n}} \frac{z^{x+n}}{x !} .
$$

If we use the recurrence [43, 18:5:12]

$$
(x)_{n+m}=(x)_{n}(x+n)_{m}, \quad n, m \in \mathbb{N}_{0},
$$

we get

$$
\nu_{n}=z^{n} \frac{(\mathbf{a})_{n}}{(\mathbf{b}+1)_{n}} \sum_{x=0}^{\infty} \frac{(\mathbf{a}+n)_{x}}{(\mathbf{b}+1+n)_{x}} \frac{z^{x}}{x !}=z^{n} \frac{(\mathbf{a})_{n}}{(\mathbf{b}+1)_{n}}{ }_{p} F_{q}\left(\begin{array}{c}
\mathbf{a}+n \\
\mathbf{b}+1+n
\end{array} ; z\right),
$$


where ${ }_{p} F_{q}$ denotes the generalized hypergeometric function defined by 44 , $16.2]$

$$
{ }_{p} F_{q}\left(\begin{array}{c}
a_{1}, \ldots, a_{p} \\
b_{1}, \ldots, b_{q}
\end{array} ; z\right)=\sum_{k=0}^{\infty} \frac{\left(a_{1}\right)_{k} \cdots\left(a_{p}\right)_{k}}{\left(b_{1}\right)_{k} \cdots\left(b_{q}\right)_{k}} \frac{z^{k}}{k !} .
$$

The moments of discrete semiclassical orthogonal polynomials of class $s \leq 1$ were studied in [18].

Remark 2 The convergence of the series (19) depends on the values of $p$ and $q$. Three different situations appear [44, 16.2]:

1. If $p<q+1$, then ${ }_{p} F_{q}$ is an entire function of $z$.

2. If $p=q+1$, then ${ }_{p} F_{q}$ is analytic inside the unit circle, $|z|<1$, and can be extended by analytic continuation to the cut plane $\mathbb{C} \backslash[1, \infty)$. Let

$$
\gamma=b_{1}+\cdots+b_{q}-\left(a_{1}+\cdots+a_{q+1}\right) .
$$

On the unit circle $|z|=1$, the series (19) is

(i) absolutely convergent if $\operatorname{Re}(\gamma)>0$,

(ii) convergent except at $z=1$ if $\operatorname{Re}(\gamma) \in(-1,0]$,

and

(iii) divergent if $\operatorname{Re}(\gamma) \leq-1$.

3. If $p>q+1$, then ${ }_{p} F_{q}$ diverges for all $z \neq 0$, up to $a_{i}=-N$, with $N \in \mathbb{N}$ for some $1 \leq i \leq p$. In this case, ${ }_{p} F_{q}$ becomes a polynomial of degree $N$.

\section{Modification of functionals}

In this section, we describe three ways in order to change the class of a linear functional. 


\subsection{Rational spectral transformations}

Let $L$ be a linear functional and $S(t)$ denote its Stieltjes transform introduced in (11). A rational spectral transformation of $S(t)$ is defined by [50]

$$
\widetilde{L}\left[\frac{1}{t-x}\right]=\widetilde{S}(t)=\frac{A(t) S(t)+B(t)}{C(t) S(t)+D(t)},
$$

where $A(t), B(t), C(t), D(t)$ are polynomials such that $A(z) D(z)-B(z) C(z) \neq$ 0 . See also [13, [23, [32, 45], [48, and [50], among others.

It was shown in [50] what are the families of semiclassical orthogonal polynomials related by spectral linear transformations (with $C=0$ ). They can be written as a composition of two basic transformations:

1. The Christoffel transformation, (see [12, [13, [14, 46]) is defined by

$$
\frac{L_{C}[r]}{\lambda_{C}}=L[(x-\omega) r(x)], \quad r \in \mathbb{C}[x],
$$

or, equivalently, by

$$
\frac{S_{C}(z)}{\lambda_{C}}=(z-\omega) S(z)-L[1]
$$

where $L(x-\omega) \neq 0, n \geq 0$, and

$$
\lambda_{C}=\frac{L_{C}[1]}{L[x-\omega]}
$$

2. The Geronimus transformation (see [15], [16], [26], [27], [32], [38]) is defined by

$$
\frac{L_{G}[r]}{\lambda_{G}}=L\left[\frac{r(x)}{x-\omega}\right]+M r(\omega), \quad r \in \mathbb{C}[x],
$$

or, equivalently, by

$$
(z-\omega) \frac{S_{G}(z)}{\lambda_{G}}=S(z)-S(\omega)+M
$$

with

$$
\lambda_{G}=\frac{L_{G}[1]}{M-S(\omega)},
$$

where we assume that $S(z)$ is analytic at $z=\omega$ and $M-S(\omega) \neq 0$. 
On the other hand, the Uvarov transformation (see [6], [33, [48]) is defined by

$$
\frac{L_{U}[r]}{\lambda_{U}}=L[r]+M r(\omega), \quad r \in \mathbb{C}[x],
$$

or by

$$
\frac{S_{U}(z)}{\lambda_{U}}=S(z)+\frac{M}{z-\omega},
$$

where $M+L[1] \neq 0$ and

$$
\lambda_{U}=\frac{L_{U}[1]}{L[1]+M}
$$

Note that since

$$
r(x)=\sum_{k=0}^{\operatorname{deg}(r)} \frac{r^{(k)}(\omega)}{k !}(x-\omega)^{k}, \quad r \in \mathbb{C}[x],
$$

we can rewrite (23) as

$$
\frac{L_{G}[r]}{\lambda_{G}}=[M-S(\omega)] r(\omega)+\sum_{k=1}^{\operatorname{deg}(r)} \frac{r^{(k)}(\omega)}{k !} L\left[(x-\omega)^{k}\right],
$$

which is well defined for all $r \in \mathbb{C}[x]$.

We denote by $L_{T_{2} T_{1}}, S_{T_{2} T_{1}}, \varrho_{T_{2} T_{1}}$, etc., the object obtained by applying the transformation $T_{1}$ followed by the transformation $T_{2}$ (in other words, by the composition $T_{2} \circ T_{1}$ ).

Let us assume that $\lambda_{U}=\lambda_{C}=\lambda_{G}=1$. If we apply a Geronimus transformation followed by a Christoffel transformation, we get

$L_{C G}[r]=L_{G}[(x-\omega) r(x)]=L\left[\frac{(x-\omega) r(x)}{x-\omega}\right]+M[(x-\omega) r(x)]_{x=\omega}=L[r]$.

On the other hand, changing the order in the composition, we obtain

$L_{G C}[r]=L_{C}\left[\frac{r(x)}{x-\omega}\right]+M r(\omega)=L\left[\frac{(x-\omega) r(x)}{x-\omega}\right]+M r(\omega)=L[r]+M r(\omega)=L_{U}[r]$.

Thus, the Christoffel and Geronimus transformations are almost inverses of each other in the sense that $L_{C G}$ is the identity and $L_{G C}$ is an Uvarov transformation. 


\subsubsection{Uvarov transformations}

Let $L$ be given by (5) and consider the linear functional $L_{U}$ defined by (25) with

$$
L_{U}[1]=L[1]+M \neq 0
$$

It follows that the moments of $L_{U}$ with respect to the basis $\left\{\phi_{n}(x)\right\}_{n \geq 0}$ are

$$
\nu_{n}^{U}=\nu_{n}+M \phi_{n}(\omega), \quad n \in \mathbb{N}_{0} .
$$

Using (26) in (12), we obtain

$$
\sigma(t+1)\left[S_{U}(t+1)-\frac{M}{t+1-\omega}\right]-\eta(t)\left[S_{U}(t)-\frac{M}{t-\omega}\right]=\xi(t) .
$$

Therefore, the Stieltjes transform of $L_{U}$ satisfies the difference equation

$$
(t+1-\omega)(t-\omega) \sigma(t+1) S_{U}(t+1)-(t+1-\omega)(t-\omega) \eta(t) S_{U}(t)=\xi_{U}(t),
$$

where

$$
\xi_{U}(t)=(t-\omega)(t-\omega+1) \xi(t)+M[(t-\omega) \sigma(t+1)-(t-\omega+1) \eta(t)]
$$

and the modified weight function $\varrho_{U}(x)$ satisfies the Pearson equation

$$
\frac{\varrho_{U}(x+1)}{\varrho_{U}(x)}=\frac{(x-\omega)(x+1-\omega) \eta(x)}{(x-\omega)(x+1-\omega) \sigma(x+1)} .
$$

We conclude that if $L$ was of class $s$, then $L_{U}$ will be of class at most $s+2$.

Notice that we have two special cases to consider:

1. If $\sigma(\omega)=0$ and $\eta(\omega) \neq 0$, with $\sigma(x)=(x-\omega) \sigma_{1}(x)$, then

$$
\begin{gathered}
\frac{\varrho_{U}(x+1)}{\varrho_{U}(x)}=\frac{(x-\omega)(x+1-\omega) \eta(x)}{(x-\omega)(x+1-\omega) \sigma(x+1)}=\frac{(x-\omega) \eta(x)}{(x-\omega) \sigma(x+1)}, \\
\sigma(t+1) S_{U}(t+1)-\eta(t) S_{U}(t)=\xi(t)+M\left[\sigma_{1}(t+1)-\frac{\eta(t)}{t-\omega}\right],
\end{gathered}
$$

and $L_{U}$ will be of class $s+1$. 
2. If $\sigma(\omega) \neq 0$ and $\eta(\omega)=0$, with $\eta(x)=(x-\omega) \eta_{1}(x)$, then

$$
\begin{gathered}
\frac{\varrho_{U}(x+1)}{\varrho_{U}(x)}=\frac{(x-\omega)(x+1-\omega) \eta(x)}{(x-\omega)(x+1-\omega) \sigma(x+1)}=\frac{(x+1-\omega) \eta(x)}{(x+1-\omega) \sigma(x+1)}, \\
\sigma(t+1) S_{U}(t+1)-\eta(t) S_{U}(t)=\xi(t)+M\left[\frac{\sigma(t+1)}{t+1-\omega}-\eta_{1}(t)\right]
\end{gathered}
$$

and $L_{U}$ will be of class $s+1$.

Perturbations of discrete semiclassical functionals by Dirac masses were studied in [2], 6], [24], [28], as well as in [3], 4], and [5].

\subsubsection{Christoffel transformations}

Let $L$ be given by (15), and consider the functional $L_{C}$ defined by (21), with

$$
L_{C}[1]=L[x-\omega] \neq 0 .
$$

It follows that the moments of $L_{C}$ with respect to the basis $\left\{\phi_{n}(x)\right\}_{n \geq 0}$ are

$$
\nu_{n}^{C}=\nu_{n+1}+(n-\omega) \nu_{n}, \quad n \in \mathbb{N}_{0}
$$

since we see from (14) that

$$
\phi_{n+1}(x)=(x-n) \phi_{n}(x)=(x-\omega) \phi_{n}(x)+(\omega-n) \phi_{n}(x) .
$$

Using (22) in (12), we obtain

$$
\sigma(t+1) \frac{S_{C}(t+1)+\nu_{0}}{t+1-\omega}-\eta(t) \frac{S_{C}(t)+\nu_{0}}{t-\omega}=\xi(t) .
$$

Therefore, the Stieltjes transform of $L_{C}$ satisfies the difference equation

$$
(t-\omega) \sigma(t+1) S_{C}(t+1)-(t+1-\omega) \eta(t) S_{C}(t)=\xi_{C}(t),
$$

where

$$
\xi_{C}(t)=(t-\omega)(t-\omega+1) \xi(t)-[(t-\omega) \sigma(t+1)-(t-\omega+1) \eta(t)] \nu_{0},
$$

and the modified weight function $\varrho_{C}(x)$ satisfies the Pearson equation

$$
\frac{\varrho_{C}(x+1)}{\varrho_{C}(x)}=\frac{(x+1-\omega) \eta(x)}{(x-\omega) \sigma(x+1)} .
$$

We conclude that if $L$ was of class $s$, then $L_{C}$ will be of class at most $s+1$.

Note that we have two special cases to consider: 
1. If $\sigma(\omega)=0$ and $\eta(\omega) \neq 0$, with $\sigma(x)=(x-\omega) \sigma_{1}(x)$, then

$$
\frac{\varrho_{C}(x+1)}{\varrho_{C}(x)}=\frac{\eta(x)}{(x-\omega) \sigma_{1}(x+1)},
$$

$(t-\omega) \sigma_{1}(t+1) S_{C}(t+1)-\eta(t) S_{C}(t)=(t-\omega) \xi(t)-\left[(t-\omega) \sigma_{1}(t+1)-\eta(t)\right] \nu_{0}$,

and $L_{C}$ could be of class $s$.

2. If $\sigma(\omega) \neq 0$ and $\eta(\omega)=0$, with $\eta(x)=(x-\omega) \eta_{1}(x)$, then

$$
\frac{\varrho_{C}(x+1)}{\varrho_{C}(x)}=\frac{(x+1-\omega) \eta_{1}(x)}{\sigma(x+1)},
$$

$\sigma(t+1) S_{C}(t+1)-(t+1-\omega) \eta_{1}(t) S_{C}(t)=(t-\omega+1) \xi(t)-\left[\sigma(t+1)-(t-\omega+1) \eta_{1}(t)\right]$ and $L_{C}$ could be of class $s$.

Christoffel transformations in the discrete case have been studied in [46].

\subsubsection{Geronimus transformations}

Let $L$ be given by (5), and consider the functional $L_{G}$ defined by (23), with

$$
L_{G}[1]=M-S(\omega) \neq 0 .
$$

Since

$$
S(\omega)=\sum_{x=0}^{\infty} \frac{1}{\omega-x} \frac{(\mathbf{a})_{x}}{(\mathbf{b}+1)_{x}} \frac{z^{x}}{x !}
$$

is a meromorphic function, we need $\omega \notin \mathbb{N}_{0}$ for $L_{G}$ [1] to be well defined.

It follows from (23) that the moments of $L_{G}$ with respect to the basis $\left\{\phi_{n}(x)\right\}_{n \geq 0}$ satisfy

$$
\nu_{n+1}^{G}+(n-\omega) \nu_{n}^{G}=\nu_{n}, \quad n \in \mathbb{N}_{0},
$$

since from (33) we see that

$$
L_{G}\left[\phi_{n+1}-(\omega-n) \phi_{n}\right]=L_{G}\left[(x-\omega) \phi_{n}(x)\right]=L\left[\phi_{n}\right] .
$$

As it is well known, the general solution of the initial value problem

$$
y_{n+1}=c_{n} y_{n}+g_{n}, \quad y_{n_{0}}=y_{0},
$$


is $[20,1.2 .4]$

$$
y_{n}=y_{0} \prod_{j=n_{0}}^{n-1} c_{j}+\sum_{k=n_{0}}^{n-1}\left(g_{k} \prod_{j=k+1}^{n-1} c_{j}\right) .
$$

Solving (34) with $\nu_{0}^{G}=M-S(\omega)$, we get

$$
\nu_{n}^{G}=\phi_{n}(\omega)\left[\nu_{0}^{G}+\sum_{k=0}^{n-1} \frac{\nu_{k}}{\phi_{k+1}(\omega)}\right],
$$

since from (14) we have

$$
\prod_{j=0}^{n-1}(\omega-j)=\phi_{n}(\omega), \quad \prod_{j=k+1}^{n-1}(\omega-j)=\frac{\phi_{n}(\omega)}{\phi_{k+1}(\omega)} .
$$

Replacing (24) in (12), we obtain

$$
\sigma(t+1)\left[(t+1-\omega) S_{G}(t+1)-\nu_{0}^{G}\right]-\eta(t)\left[(t-\omega) S_{G}(t)-\nu_{0}^{G}\right]=\xi(t) .
$$

Therefore, the Stieltjes transform of $L_{G}$ satisfies the difference equation

$$
(t+1-\omega) \sigma(t+1) S_{G}(t+1)-(t-\omega) \eta(t) S_{G}(t)=\xi_{G}(t),
$$

where

$$
\xi_{G}(t)=\xi(t)+[\sigma(t+1)-\eta(t)] \nu_{0}^{G},
$$

and the modified weight function $\varrho_{G}(x)$ satisfies the Pearson equation

$$
\frac{\varrho_{G}(x+1)}{\varrho_{G}(x)}=\frac{(x-\omega) \eta(x)}{(x+1-\omega) \sigma(x+1)} .
$$

We conclude that if $L$ was of class $s$, then $L_{G}$ will be of class at most $s+1$.

Remark 3 For all these transformations, it is understood that

$$
\begin{aligned}
& \eta(\omega)=0 \Rightarrow \frac{\eta(x)}{x-\omega} \times(x-\omega)=\eta(x), \\
& \sigma(\omega)=0 \Rightarrow \frac{x+1-\omega}{\sigma(x+1)} \times \frac{1}{x+1-\omega}=\frac{1}{\sigma(x+1)} .
\end{aligned}
$$


Thus, if we write

$$
\frac{\varrho_{G}(x+1)}{\varrho_{G}(x)}=\frac{(x-\omega) \eta(x)}{(x+1-\omega) \sigma(x+1)}=\frac{\eta_{G}(x)}{\sigma_{G}(x+1)},
$$

then clearly $\eta_{G}(\omega)=0=\sigma_{G}(\omega)$. Hence,

$$
\frac{\varrho_{C G}(x+1)}{\varrho_{C G}(x)}=\frac{\eta_{G}(x)}{\sigma_{G}(x+1)} \times \frac{x-\omega}{x+1-\omega}=\frac{\eta(x)}{\sigma(x+1)}=\frac{\varrho(x+1)}{\varrho(x)},
$$

in agreement with (27).

On the other hand, if we write

$$
\frac{\varrho_{C}(x+1)}{\varrho_{C}(x)}=\frac{(x+1-\omega) \eta(x)}{(x-\omega) \sigma(x+1)}=\frac{\eta_{C}(x)}{\sigma_{C}(x+1)},
$$

then $\eta_{G}(\omega) \sigma_{G}(\omega) \neq 0$, and therefore

$$
\begin{aligned}
\frac{\varrho_{G C}(x+1)}{\varrho_{G C}(x)} & =\frac{\eta_{C}(x)}{\sigma_{C}(x+1)} \times \frac{x+1-\omega}{x-\omega} \\
& =\frac{(x-\omega)(x+1-\omega) \eta(x)}{(x-\omega)(x+1-\omega) \sigma(x+1)}=\frac{\varrho_{U}(x+1)}{\varrho_{U}(x)},
\end{aligned}
$$

in agreement with (28).

\subsection{Truncated linear functionals}

Let $N \in \mathbb{N}_{0}$ and define the truncated functional $L_{T}$ of the linear functional $L$ by

$$
L_{T}[r]=\sum_{x=0}^{N} r(x) \varrho(x), \quad r \in \mathbb{C}[x],
$$

where $\varrho(x)$ satisfies (7) with $\eta(N) \neq 0$. We define the truncated weight function $\varrho_{T}(x)$ by

$$
\varrho_{T}(x)=\varrho(x)[1-H(x-N)],
$$

where $H(x)$ is the Heaviside function [44, 1.16(iv)] defined by

$$
H(x)=\left\{\begin{array}{ll}
1, & x>0 \\
0, & x \leq 0
\end{array} .\right.
$$


Multiplying (36) by $\frac{1}{(N-x) !}$, we obtain

$$
\frac{1}{(N-x) !} \varrho_{T}(x)=\frac{1}{(N-x) !} \varrho(x), \quad x \in \mathbb{N}_{0}
$$

Using (37) in (7), we get

$$
(N-x) \frac{\varrho_{T}(x+1)}{\varrho_{T}(x)}=\frac{\frac{1}{(N-x-1) !} \varrho_{T}(x+1)}{\frac{1}{(N-x) !} \varrho_{T}(x)}=\frac{\frac{1}{(N-x-1) !} \varrho(x+1)}{\frac{1}{(N-x) !} \varrho(x)}=(N-x) \frac{\eta(x)}{\sigma(x+1)} .
$$

We conclude that the modified weight function $\varrho_{T}(x)$ satisfies the Pearson equation

$$
\frac{\varrho_{T}(x+1)}{\varrho_{T}(x)}=\frac{\eta(x)(x-N)}{\sigma(x+1)(x-N)},
$$

and $L_{T}$ will be of class $s+1$.

The moments of $L_{T}$ with respect to the basis $\left\{\phi_{n}(x)\right\}_{n \geq 0}$ are

$$
\nu_{n}^{T}(z)=\sum_{x=0}^{N} \phi_{n}(x) \frac{(\mathbf{a})_{x}}{(\mathbf{b}+1)_{x}} \frac{z^{x}}{x !}, \quad n \in \mathbb{N}_{0} .
$$

A similar calculation leading to (18), gives

$$
\nu_{n}^{T}(z)=z^{n} \frac{(\mathbf{a})_{n}}{(\mathbf{b}+1)_{n}} \sum_{x=0}^{N-n} \frac{(\mathbf{a}+n)_{x}}{(\mathbf{b}+1+n)_{x}} \frac{z^{x}}{x !}, \quad n \in \mathbb{N}_{0} .
$$

Using the identity [44, 16.2.4]

$\sum_{x=0}^{K} \frac{(\mathbf{a})_{x}}{(\mathbf{b}+1)_{x}} \frac{z^{x}}{x !}=\frac{(\mathbf{a})_{K}}{(\mathbf{b}+1)_{K}} \frac{z^{K}}{K !}{ }_{q+2} F_{p}\left[\begin{array}{c}-K, 1,-K-\mathbf{b} \\ 1-K-\mathbf{a}\end{array} ; \frac{(-1)^{p+q+1}}{z}\right], \quad K \in \mathbb{N}$,

we can rewrite (39) as

$$
\nu_{n}^{T}(z)=\frac{(\mathbf{a})_{N}}{(\mathbf{b}+1)_{N}} \frac{z^{N}}{(N-n) !}{ }^{q+2} F_{p}\left[\begin{array}{c}
n-N, 1,-N-\mathbf{b} \\
1-N-\mathbf{a}
\end{array} ; \frac{(-1)^{p+q+1}}{z}\right] .
$$




\subsection{Symmetrized functionals}

Let $\varrho(x)$ be given by $(\underline{6})$ and $m \in \mathbb{N}$. We consider the symmetric weight function $\varrho_{\varsigma}(x)$ defined by

$$
\varrho_{\varsigma}(x)=C \varrho(x+m) \varrho(-x+m) \text {, }
$$

where $C$ is a constant factor to be determined. Replacing (7) in (41), we get

$\frac{\varrho_{\varsigma}(x+1)}{\varrho_{\varsigma}(x)}=\frac{\varrho(x+1+m)}{\varrho(x+m)} \frac{\varrho(-x-1+m)}{\varrho(-x+m)}=\frac{\eta(x+m)}{\sigma(x+m+1)} \frac{\sigma(-x+m)}{\eta(-x-1+m)}$.

Therefore,

$$
\frac{\varrho_{\varsigma}(x+1)}{\varrho_{\varsigma}(x)}=\frac{x+m+\mathbf{a}}{(x+m+\mathbf{b}+1)(x+m+1)} \frac{(m+\mathbf{b}-x)(m-x)}{m+\mathbf{a}-x-1},
$$

or

$$
\frac{\varrho_{\varsigma}(x+1)}{\varrho_{\varsigma}(x)}=\frac{(-1)^{p+q+1}(x+m+\mathbf{a})(x-m-\mathbf{b})(x-m)}{(x+1-m-\mathbf{a})(x+1+m+\mathbf{b})(x+1+m)} .
$$

Note that, from (42), we have $\varrho_{\varsigma}(x)=\varrho_{1}(x+m)$, with

$$
\frac{\varrho_{1}(x+1)}{\varrho_{1}(x)}=\frac{(-1)^{p+q+1}(x+\mathbf{a})(x-2 m-\mathbf{b})(x-2 m)}{(x+1-2 m-\mathbf{a})(x+1+\mathbf{b})(x+1)} .
$$

If we write $N=2 m$, then

$$
\frac{\varrho_{1}(x+1)}{\varrho_{1}(x)}=\frac{(-1)^{p+q+1}(x+\mathbf{a})(x-N-\mathbf{b})(x-N)}{(x+1-N-\mathbf{a})(x+1+\mathbf{b})(x+1)},
$$

and it follows that $\varrho_{1}(x)$ (and $\varrho_{\varsigma}$ ) is of class $s_{1}$ with

$$
s_{1}=\left\{\begin{array}{c}
p+q, \quad \text { if } q+p \text { is even } \\
p+q-1, \quad \text { if } q+p \text { is odd }
\end{array} .\right.
$$

If $\mathbf{a}=\left(\mathbf{a}_{1},-N\right)$, then

$$
\frac{\varrho_{1}(x+1)}{\varrho_{1}(x)}=\frac{(-1)^{q+1+p}\left(x+\mathbf{a}_{1}\right)}{(x+1+\mathbf{b})(x+1)} \frac{x-N}{x+1} \frac{(x-N-\mathbf{b})(x-N)}{x+1-N-\mathbf{a}_{1}},
$$


and since the term $\frac{x-N}{x+1}$ is repeated, we consider the reduced Pearson equation

$$
\begin{aligned}
\frac{\varrho_{2}(x+1)}{\varrho_{2}(x)} & =\frac{(-1)^{q+1+p}\left(x+\mathbf{a}_{1}\right)}{(x+1+\mathbf{b})(x+1)} \frac{(x-N-\mathbf{b})(x-N)}{x+1-N-\mathbf{a}_{1}} \\
& =\frac{(-1)^{q+1+p}(x+\mathbf{a})}{(x+1+\mathbf{b})} \frac{(x-N-\mathbf{b})}{x+1-N-\mathbf{a}}
\end{aligned}
$$

and $\varrho_{\varsigma}(x)=\varrho_{2}(x+m)$. In this case $\varrho_{2}(x)\left(\right.$ and $\left.\varrho_{\varsigma}\right)$ is of class $s_{2}$ with

$$
s_{2}=\left\{\begin{array}{cc}
p+q-1, & \text { if } q+p \text { is even } \\
p+q-2, & \text { if } q+p \text { is odd }
\end{array} .\right.
$$

As a conclusion, if $\varrho(x)$ satisfies (7), then we have $\varrho_{\varsigma}(x)=\rho(x+m)$ where

$$
\rho(x)=\left\{\begin{array}{cc}
\frac{(\mathbf{a})_{x}}{(\mathbf{b}+1)_{x}} \frac{\left(z_{0}\right)^{x}}{x !} \frac{(-N)_{x}(-N-\mathbf{b})_{x}}{(1-N-\mathbf{a})_{x}}, \quad \eta(N) \neq 0, \\
\frac{(\mathbf{a})_{x}}{(\mathbf{b}+1)_{x}}\left(z_{0}\right)^{x} \frac{(-N-\mathbf{b})_{x}}{(1-N-\mathbf{a})_{x}}, \quad \eta(N)=0,
\end{array}\right.
$$

and

$$
z_{0}=(-1)^{p+q+1}, \quad N=2 m .
$$

We define the symmetrized functional $L_{\varsigma}$ of $L_{\rho}$ by

$$
L_{\varsigma}[r]=\sum_{x=-m}^{m} r(x) \varrho_{\varsigma}(x)=\sum_{x=0}^{N} r(x-m) \rho(x)=L_{\rho}[r(x-m)] .
$$

Hence, the moments of $L_{\varsigma}$ on the basis

$$
\bar{\phi}_{n}(x)=\phi_{n}(x+m), \quad n \in \mathbb{N}_{0},
$$

are

$$
\nu_{n}^{\varsigma}=\sum_{x=-m}^{m} \bar{\phi}_{n}(x) \varrho_{\varsigma}(x)=\sum_{x=0}^{N} \phi_{n}(x) \rho(x)=L_{\rho}\left[\phi_{n}\right], \quad n \in \mathbb{N}_{0},
$$

and the Stieltjes transform of $L_{\varsigma}$ is

$$
S_{\varsigma}(t)=\sum_{x=-m}^{m} \frac{\varrho_{\varsigma}(x)}{t-x}=\sum_{x=0}^{N} \frac{\rho(x)}{t+m-x}=S_{\rho}(t+m) .
$$


It follows that if the Stieltjes transform of $L_{\rho}$ satisfies the difference equation

$$
\sigma_{\rho}(t+1) S_{\rho}(t+1)-\eta_{\rho}(t) S_{\rho}(t)=\xi_{\rho}(t)
$$

then

$$
\sigma_{\rho}(t+m+1) S_{\rho}(t+m+1)-\eta_{\rho}(t+m) S_{\rho}(t+m)=\xi_{\rho}(t+m) .
$$

Therefore

$$
\sigma_{\varsigma}(t+1) S_{\varsigma}(t+1)-\eta_{\varsigma}(t) S_{\varsigma}(t)=\xi_{\rho}(t+m)
$$

since

$$
\frac{\eta_{\varsigma}(x)}{\sigma_{\varsigma}(x+1)}=\frac{\varrho_{\varsigma}(x+1)}{\varrho_{\varsigma}(x)}=\frac{\rho(x+m+1)}{\rho(x+m)}=\frac{\eta_{\rho}(x+m)}{\sigma_{\rho}(x+m+1)} .
$$

Symmetric orthogonal polynomials of a discrete variable were studied in [7], [8], [40], [41].

\subsection{Summary}

Let's list all the transformations that we will use in the sequel. For simplicity, we will not consider compositions of them.

1. Uvarov transformation: $s \rightarrow s+2$

$$
\frac{\varrho_{U}(x+1)}{\varrho_{U}(x)}=\frac{\eta(x)}{\sigma(x+1)} \times \frac{(x-\omega)(x+1-\omega)}{(x-\omega)(x+1-\omega)}, \quad \eta(\omega) \sigma(\omega) \neq 0 .
$$

2. Reduced Uvarov transformation: $s \rightarrow s+1$

(a)

$$
\frac{\varrho_{U}(x+1)}{\varrho_{U}(x)}=\frac{\eta(x)}{\sigma(x+1)} \times \frac{x+1-\omega}{x+1-\omega}, \quad \eta(\omega)=0
$$

(b)

$$
\frac{\varrho_{U}(x+1)}{\varrho_{U}(x)}=\frac{\eta(x)}{\sigma(x+1)} \times \frac{x-\omega}{x-\omega}, \quad \sigma(\omega)=0 .
$$

3. Christoffel transformation: $s \rightarrow s+1$

$$
\frac{\varrho_{C}(x+1)}{\varrho_{C}(x)}=\frac{\eta(x)}{\sigma(x+1)} \times \frac{x+1-\omega}{x-\omega}, \quad \eta(\omega) \sigma(\omega) \neq 0 .
$$


4. Geronimus transformation: $s \rightarrow s+1$

$$
\frac{\varrho_{G}(x+1)}{\varrho_{G}(x)}=\frac{\eta(x)}{\sigma(x+1)} \times \frac{x-\omega}{x+1-\omega}, \quad \eta(\omega-1) \sigma(\omega+1) \neq 0 .
$$

5. Truncation at $x=N: s \rightarrow s+1$

$$
\frac{\varrho_{T}(x+1)}{\varrho_{T}(x)}=\frac{\eta(x)}{\sigma(x+1)} \times \frac{x-N}{x-N}, \quad \eta(N) \neq 0 .
$$

6. Symmetrization on the interval $[-m, m]$ :

(a)

$$
\frac{\varrho_{\varsigma}(x+1)}{\varrho_{\varsigma}(x)}=\frac{\eta(m+x)}{\sigma(m+x+1)} \times \frac{\sigma(m-x)}{\eta(m-x-1)}, \quad \eta(2 m) \neq 0 .
$$

(b)

$$
\frac{\varrho_{\varsigma}(x+1)}{\varrho_{\varsigma}(x)}=\frac{\eta(m+x)}{\sigma(m+x+1)} \times \frac{\sigma_{1}(m-x)}{\eta_{1}(m-x-1)}, \quad \eta(2 m)=0,
$$

where

$$
\eta(x)=(x-2 m) \eta_{1}(x), \quad \sigma(x)=x \sigma_{1}(x) .
$$

\section{Semiclassical polynomials of class 0 (classical polynomials)}

In this section, we consider the families of discrete classical polynomials. We have 3 main cases, corresponding to $(p, q)=(0,0),(1,0),(2,1)$. There are also 3 symmetrized subcases.

We use the notation $(p, q ; N)$ to denote the family such that one of the parameters in the numerator is a non-negative integer, and $(p, q ; N, 1)$ if in addition the value of $z$ is equal to 1 .

For each polynomial, we list the linear functional, the Pearson equation satisfied by the weight function, the moments computed from (18), and the difference equation satisfied by the Stieltjes transform, using (13) and (44). 


\section{$4.1(0,0)$ : Charlier polynomials}

Linear functional

$$
L[r]=\sum_{x=0}^{\infty} r(x) \frac{z^{x}}{x !}
$$

Pearson equation

$$
\frac{\varrho(x+1)}{\varrho(x)}=\frac{z}{x+1} .
$$

Moments

$$
\nu_{n}(z)=z_{0}^{n} F_{0}\left[\begin{array}{l}
- \\
-
\end{array}\right]=z^{n} e^{z}, \quad n \in \mathbb{N}_{0} .
$$

Stieltjes transform difference equation

$$
(t+1) S(t+1)-z S(t)=\nu_{0}
$$

\section{$4.2(1,0)$ : Meixner polynomials}

Linear functional

$$
L[r]=\sum_{x=0}^{\infty} r(x)(a)_{x} \frac{z^{x}}{x !}
$$

Pearson equation

$$
\frac{\varrho(x+1)}{\varrho(x)}=\frac{z(x+a)}{x+1} .
$$

Moments

$$
\nu_{n}(z)=z^{n}(a)_{n}{ }_{1} F_{0}\left[\begin{array}{c}
a+n \\
-
\end{array} ; z\right]=z^{n}(a)_{n}(1-z)^{-a-n}, \quad n \in \mathbb{N}_{0},
$$

where we choose the principal branch $z \in \mathbb{C} \backslash[1, \infty)$.

Stieltjes transform difference equation

$$
(t+1) S(t+1)-z(t+a) S(t)=(1-z) \nu_{0} .
$$




\section{$4.3(1,0 ; N)$ : Krawtchouk polynomials}

These polynomials are a particular case of the Meixner polynomials, with $-a=N \in \mathbb{N}$.

Linear functional

$$
L[r]=\sum_{x=0}^{N} r(x)(-N)_{x} \frac{z^{x}}{x !} .
$$

Pearson equation

$$
\frac{\varrho(x+1)}{\varrho(x)}=\frac{z(x-N)}{x+1} .
$$

Moments

$$
\nu_{n}(z)=z^{n}(-N)_{n}(1-z)^{N-n}, \quad z \neq 1, \quad n \in \mathbb{N}_{0} .
$$

Stieltjes transform difference equation

$$
(t+1) S(t+1)-z(t-N) S(t)=(1-z) \nu_{0} .
$$

Remark 4 Let's consider the symmetrized Krawtchouk polynomials. Since $\eta(N)=0$, we use (45) and obtain

$$
\frac{\varrho_{\varsigma}(x+1)}{\varrho_{\varsigma}(x)}=\frac{z(x-m)}{x+m+1} \frac{(x+m+1)(-x+m)}{(x-m) z(-x-1-m)}=\frac{x-m}{x+m+1} .
$$

Hence, the symmetrized Krawtchouk polynomials are shifted Krawtchouk polynomials with $z=1$. But from (43) and (46) we see that

$$
\nu_{n}^{\varsigma}=\nu_{n}(1)=0, \quad n \in \mathbb{N}_{0},
$$

and, therefore, we need to discard this example.

\subsubsection{Symmetrized Charlier polynomials}

Special values

$$
N \rightarrow 2 m, \quad z \rightarrow-1, \quad x \rightarrow x+m .
$$

Weight function

$$
\begin{aligned}
\varrho_{\varsigma}(x) & =\varrho(x+m)=(-2 m)_{x+m} \frac{(-1)^{x+m}}{(x+m) !} \\
& =(-2 m)_{m} \frac{(-1)^{m}}{m !} \frac{(-m)_{x}}{(m+1)_{x}}(-1)^{x}=\left(\begin{array}{c}
2 m \\
m
\end{array}\right) \frac{(-m)_{x}}{(m+1)_{x}}(-1)^{x} .
\end{aligned}
$$


Linear functional

$$
L_{\varsigma}[r]=\left(\begin{array}{c}
2 m \\
m
\end{array}\right) \sum_{x=-m}^{m} r(x) \frac{(-m)_{x}}{(m+1)_{x}}(-1)^{x} .
$$

Pearson equation

$$
\frac{\varrho_{\varsigma}(x+1)}{\varrho_{\varsigma}(x)}=\frac{-(x-m)}{x+m+1} .
$$

Moments on the basis $\left\{\bar{\phi}_{n}(x)\right\}_{n \geq 0}$

$$
\nu_{n}^{\varsigma}=L_{\varsigma}[\phi(x+m)]=(-1)^{n}(-2 m)_{n} 2^{2 m-n}=\nu_{n}(-1), \quad n \in \mathbb{N}_{0},
$$

where $\nu_{n}(z)$ are the moments of the Krawtchouk polynomials defined in (46).

Stieltjes transform difference equation

$$
(t+m+1) S_{\varsigma}(t+1)+(t-m) S_{\varsigma}(t)=2 \nu_{0}^{\varsigma}
$$

Remark 5 These polynomials were studied in [8]. The Pearson equation (47) is the same as equation (11) in that paper, with $m=c$. The authors used the weight function

$$
\rho(x)=\frac{1}{\Gamma(x+m+1) \Gamma(-x+m+1)}=\frac{1}{(m !)^{2}} \frac{(-m)_{x}}{(m+1)_{x}}(-1)^{x},
$$

and, therefore,

$$
\varrho_{\varsigma}(x)=(2 m) ! \rho(x) .
$$

The moments (48) are the same as those appearing in equation (15), if we use (15) and write

$$
\nu_{n}^{\varsigma}=(-1)^{n}(-2 m)_{n} 2^{2 m-n}=2^{2 m-n} \phi_{n}(2 m),
$$

after taking the scaling (49) into account.

\section{$4.4(2,1 ; N, 1)$ : Hahn polynomials}

Linear functional

$$
L[r]=\sum_{x=0}^{N} r(x) \frac{(a)_{x}(-N)_{x}}{(b+1)_{x}} \frac{1}{x !}
$$


Pearson equation

$$
\frac{\eta(x)}{\sigma(x+1)}=\frac{\varrho(x+1)}{\varrho(x)}=\frac{(x+a)(x-N)}{(x+b+1)(x+1)} .
$$

Moments

$$
\nu_{n}=\frac{(-N)_{n}(a)_{n}}{(b+1)_{n}}{ }_{2} F_{1}\left(\begin{array}{c}
-N+n, a+n \\
b+1+n
\end{array} ; 1\right), n \in \mathbb{N}_{0} .
$$

Using the Chu-Vandermonde Identity [44, 15.4.24],

$$
{ }_{2} F_{1}\left(\begin{array}{cc}
-n, b & ; 1 \\
c & ;
\end{array}\right)=\frac{(c-b)_{n}}{(c)_{n}},
$$

we get

$$
\nu_{n}=\frac{(-N)_{n}(a)_{n}}{(b+1)_{n}} \frac{(b+1-a)_{N-n}}{(b+1+n)_{N-n}}, \quad n \in \mathbb{N}_{0} .
$$

Stieltjes transform difference equation

$$
(t+1)(t+b+1) S(t+1)-(t-N)(t+a) S(t)=(b+1-a+N) \nu_{0} \text {. }
$$

\subsubsection{Symmetrized Meixner polynomials}

Special values

$$
b \rightarrow-N-a, \quad N \rightarrow 2 m, \quad x \rightarrow x+m .
$$

Linear functional

$$
L_{\varsigma}[r]=\left(\begin{array}{c}
2 m \\
m
\end{array}\right) \frac{(a)_{m}}{(a+m)_{m}} \sum_{x=-m}^{m} r(x) \frac{(a+m)_{x}(-m)_{x}}{(-m-a+1)_{x}(m+1)_{x}} .
$$

Pearson equation

$$
\frac{\varrho_{\varsigma}(x+1)}{\varrho_{\varsigma}(x)}=\frac{(x+a+m)(x-m)}{(x+1-m-a)(x+1+m)} .
$$

Moments

$$
\nu_{n}^{\varsigma}=\frac{(-N)_{n}(a)_{n}}{(1-N-a)_{n}} \frac{(1-N-2 a)_{N-n}}{(n+1-N-a)_{N-n}}, \quad n \in \mathbb{N}_{0} .
$$

Stieltjes transform difference equation

$(t+m+1)(t-m-a+1) S_{\varsigma}(t+1)-(t-m)(t+m+a) S_{\varsigma}(t)=(1-2 a) \nu_{0}^{\varsigma}$. 
Remark 6 If we use (4) and (41) to write the weight function $\varrho_{\varsigma}(x)$ in terms of Gamma functions, we have

$\varrho_{\varsigma}(x)=\varrho_{\varsigma}(0)\left[\frac{m !}{(a)_{m}}\right]^{2} \frac{(a)_{x+m}}{(x+m) !} \frac{(a)_{-x+m}}{(-x+m) !}=\frac{(2 m) !}{(a)_{2 m}} \frac{\Gamma(x+m+a) \Gamma(-x+m+a)}{\Gamma(x+m+1) \Gamma(-x+m+1)}$.

This agrees (up to a normalization factor) with the weight function considered by the authors in [8] (equation 27), if we set $\delta_{1}=-m$ and $\delta_{2}=m+a$. Note that the condition $\delta_{2}>-\delta_{1}$ is satisfied if $a>0$ (the positive-definite case).

\subsubsection{Symmetrized generalized Charlier polynomials}

See section 5.1 for a definition of the Generalized Charlier polynomials.

Special values

$$
a \rightarrow-N-b, \quad N \rightarrow 2 m, \quad x \rightarrow x+m .
$$

Linear functional

$$
L_{\varsigma}[r]=\left(\begin{array}{c}
2 m \\
m
\end{array}\right) \frac{(b+1+m)_{m}}{(b+1)_{m}} \sum_{x=-m}^{m} r(x) \frac{(-m-b)_{x}(-m)_{x}}{(b+1+m)_{x}(m+1)_{x}} .
$$

Pearson equation

$$
\frac{\varrho_{\varsigma}(x+1)}{\varrho_{\varsigma}(x)}=\frac{(x-m-b)(x-m)}{(x+m+b+1)(x+m+1)} .
$$

Moments

$$
\nu_{n}^{\varsigma}=\frac{(-2 m)_{n}(-2 m-b)_{n}}{(b+1)_{n}} \frac{(2 m+2 b+1)_{2 m-n}}{(b+1+n)_{2 m-n}}, \quad n \in \mathbb{N}_{0} .
$$

Stieltjes transform difference equation

$$
(t+m+1)(t+m+b+1) S_{\varsigma}(t+1)-(t-m)(t-m-b) S_{\varsigma}(t)=(2 b+1+4 m) \nu_{0}^{\varsigma} .
$$

Remark 7 If we use (4) and (41) to write the weight function $\varrho_{\varsigma}(x)$ in terms of Gamma functions, we have

$$
\begin{aligned}
\varrho_{\varsigma}(x) & =\varrho_{\varsigma}(0)\left[(b+1)_{m} m !\right]^{2} \frac{1}{(b+1)_{x+m}(x+m) !} \frac{1}{(b+1)_{-x+m}(-x+m) !} \\
& =\frac{(b+1)_{2 m}(2 m) !}{\Gamma(x+m+b+1) \Gamma(x+m+1) \Gamma(-x+m+b+1) \Gamma(-x+m+1)} .
\end{aligned}
$$

This agrees (up to a normalization factor) with the weight function considered by the authors in [8] (equation 26). 


\section{Semiclassical polynomials of class 1}

In this section, we consider all families of polynomials of class 1 . We have 5 main cases, corresponding to

$$
(p, q)=(0,1),(1,1),(2,0),(2,1),(3,2) .
$$

There are also 14 subcases.

\section{$5.1(0,1)$ : Generalized Charlier polynomials}

Linear functional

$$
L[r]=\sum_{x=0}^{\infty} r(x) \frac{1}{(b+1)_{x}} \frac{z^{x}}{x !}
$$

Pearson equation

$$
\frac{\varrho(x+1)}{\varrho(x)}=\frac{z}{(x+b+1)(x+1)} .
$$

Moments

$$
\nu_{n}(z)=\frac{z^{n}}{(b+1)_{n}}{ }_{0} F_{1}\left[\begin{array}{c}
- \\
b+1+n
\end{array} ; z\right], \quad n \in \mathbb{N}_{0} .
$$

Stieltjes transform difference equation

$$
(t+1)(t+b+1) S(t+1)-z S(t)=(t+b+1) \nu_{0}+\nu_{1} .
$$

\section{$5.2(1,1)$ : Generalized Meixner polynomials}

Linear functional

$$
L[r]=\sum_{x=0}^{\infty} r(x) \frac{(a)_{x}}{(b+1)_{x}} \frac{z^{x}}{x !}
$$

Pearson equation

$$
\frac{\varrho(x+1)}{\varrho(x)}=\frac{z(x+a)}{(x+1)(x+b+1)} .
$$


Moments

$$
\nu_{n}(z)=z^{n} \frac{(a)_{n}}{(b+1)_{n}}{ }_{1} F_{1}\left[\begin{array}{c}
a+n \\
b+1+n
\end{array} ; z\right], \quad n \in \mathbb{N}_{0} .
$$

Stieltjes transform difference equation

$$
(t+1)(t+b+1) S(t+1)-z(t+a) S(t)=(t+b+1-z) \nu_{0}+\nu_{1} \text {. }
$$

\subsubsection{Reduced-Uvarov Charlier polynomials}

Let $\omega=0$.

Special values

$$
a \rightarrow 0, \quad b \rightarrow-1
$$

Linear functional

$$
L_{U}[r]=\sum_{x=0}^{\infty} r(x) \frac{z^{x}}{x !}+M r(0) .
$$

Pearson equation

$$
\frac{\varrho_{U}(x+1)}{\varrho_{U}(x)}=\frac{z x}{(x+1) x}
$$

Moments

$$
\nu_{n}^{U}(z)=z^{n} e^{z}+M \phi_{n}(0), \quad n \in \mathbb{N}_{0} .
$$

Stieltjes transform difference equation

$$
t\left[(t+1) S_{U}(t+1)-z S_{U}(t)\right]=(t-z) \nu_{0}^{U}+\nu_{1}^{U}
$$

Since

$$
(t-z) \nu_{0}^{U}+\nu_{1}^{U}=(t-z)\left(e^{z}+M\right)+z e^{z}=t \nu_{0}+M(t-z),
$$

where $\nu_{0}=e^{z}$ is the first moment of the Charlier polynomials, we can rewrite (52) in the $M$-dependent form

$$
(t+1) S_{U}(t+1)-z S_{U}(t)=\nu_{0}+M\left(1-\frac{z}{t}\right),
$$

which agrees with (31). 


\subsubsection{Christoffel Charlier polynomials}

Let $\omega \notin\{0, z\}$.

Special values

$$
a \rightarrow-\omega+1, \quad b \rightarrow-\omega-1 .
$$

Linear functional

$$
L_{C}[r]=\sum_{x=0}^{\infty} r(x)(x-\omega) \frac{z^{x}}{x !}
$$

Pearson equation

$$
\frac{\varrho_{C}(x+1)}{\varrho_{C}(x)}=\frac{z(x+1-\omega)}{(x+1)(x-\omega)} .
$$

Moments

$$
\nu_{n}^{C}(z)=\nu_{n+1}+(n-\omega) \nu_{n}=(n+z-\omega) z^{n} e^{z}, \quad n \in \mathbb{N}_{0} .
$$

Stieltjes transform difference equation

$(t-\omega)(t+1) S_{C}(t+1)-(t+1-\omega) z S_{C}(t)=\nu_{0}\left[(z-\omega) t+\left(\omega^{2}-z \omega+z\right)\right]$.

Note that if we use (53), we have

$$
\nu_{0}^{C}=(z-\omega) \nu_{0}, \quad \nu_{1}^{C}=(1+z-\omega) z \nu_{0},
$$

and therefore

$$
(t-\omega)(t+1) S_{C}(t+1)-(t+1-\omega) z S_{C}(t)=(t-\omega-z) \nu_{0}^{C}+\nu_{1}^{C},
$$

which agrees with (51) as $a \rightarrow 1-\omega, \quad b \rightarrow-\omega-1$.

\subsubsection{Geronimus Charlier polynomials}

Let $\omega \notin\{-1\} \cup \mathbb{N}_{0}$.

Special values

$$
a \rightarrow-\omega, \quad b \rightarrow-\omega .
$$

Linear functional

$$
L_{G}[r]=\sum_{x=0}^{\infty} \frac{r(x)}{x-\omega} \frac{z^{x}}{x !}+M r(\omega) .
$$


Pearson equation

$$
\frac{\varrho_{G}(x+1)}{\varrho_{G}(x)}=\frac{z(x-\omega)}{(x+1)(x+1-\omega)} .
$$

Moments

$$
\nu_{n}^{G}(z)=\phi_{n}(\omega)\left[M-S(\omega)+e^{z} \sum_{k=0}^{n-1} \frac{z^{k}}{\phi_{k+1}(\omega)}\right], \quad n \in \mathbb{N}_{0} .
$$

Stieltjes transform difference equation

$$
(t+1-\omega) \sigma(t+1) S_{G}(t+1)-(t-\omega) \eta(t) S_{G}(t)=\nu_{0}+\nu_{0}^{G}(t+1-z) \text {. }
$$

Note that if we use (34), we have

$$
\nu_{1}^{G}-\omega \nu_{0}^{G}=\nu_{0}
$$

and therefore we can write

$$
(t+1)(t+1-\omega) S_{G}(t+1)-z(t-\omega) S_{G}(t)=(t+1-\omega-z) \nu_{0}^{G}+\nu_{1}^{G},
$$

which agrees with (51) as $a \rightarrow-\omega, \quad b \rightarrow-\omega$.

\subsubsection{Truncated Charlier polynomials}

Special values

$$
a \rightarrow-N, \quad b \rightarrow-N-1
$$

Linear functional

$$
L_{T}[r]=\sum_{x=0}^{N} r(x) \frac{z^{x}}{x !}
$$

Pearson equation

$$
\frac{\varrho_{T}(x+1)}{\varrho_{T}(x)}=\frac{z(x-N)}{(x+1)(x-N)} .
$$

Moments

$$
\nu_{n}^{T}(z)=\frac{z^{N}}{(N-n) !}{ }_{2} F_{0}\left[\begin{array}{c}
n-N, 1 \\
-
\end{array} ;-\frac{1}{z}\right], \quad n \in \mathbb{N}_{0} .
$$

Stieltjes transform difference equation

$$
(t-N)\left[(t+1) S_{T}(t+1)-z S_{T}(t)\right]=(t-N-z) \nu_{0}^{T}+\nu_{1}^{T} .
$$

Remark 8 The performance of the modified Chebyshev algorithm used to compute the moments of these polynomials was studied in [25] (example 4.3). 


\section{$5.3(2,0 ; N)$ : Generalized Krawtchouk polynomials}

Linear functional

$$
L[r]=\sum_{x=0}^{N} r(x)(a)_{x}(-N)_{x} \frac{z^{x}}{x !}
$$

Pearson equation

$$
\frac{\varrho(x+1)}{\varrho(x)}=\frac{z(x+a)(x-N)}{x+1} .
$$

Moments

$$
\nu_{n}(z)=z^{n}(-N)_{n}(a)_{n}{ }_{2} F_{0}\left[\begin{array}{c}
-N+n, a+n \\
-
\end{array}\right], \quad n \in \mathbb{N}_{0} .
$$

Stieltjes transform difference equation

$$
(t+1) S(t+1)-z(t+a)(t-N) S(t)=[1-(t+a-N) z] \nu_{0}-z \nu_{1}
$$

\section{$5.4(2,1)$ : Generalized Hahn polynomials of type I}

Linear functional

$$
L[r]=\sum_{x=0}^{\infty} r(x) \frac{\left(a_{1}\right)_{x}\left(a_{2}\right)_{x}}{(b+1)_{x}} \frac{z^{x}}{x !}
$$

Pearson equation

$$
\frac{\varrho(x+1)}{\varrho(x)}=\frac{z\left(x+a_{1}\right)\left(x+a_{2}\right)}{(x+b+1)(x+1)} .
$$

Moments

$$
\nu_{n}(z)=z^{n} \frac{\left(a_{1}\right)_{n}\left(a_{2}\right)_{n}}{(b+1)_{n}} F_{1}\left[\begin{array}{c}
a_{1}+n, a_{2}+n \\
b+1+n
\end{array} ; z\right], \quad n \in \mathbb{N}_{0},
$$

where we choose the principal branch $z \in \mathbb{C} \backslash[1, \infty)$. Note that since

$$
b+1+n-\left(a_{1}+n+a_{2}+n\right) \leq 0, \quad n \geq b-a_{1}-a_{2}+1,
$$

$\nu_{n}(1)$ is undefined for $n \geq b-a_{1}-a_{2}+1$.

Stieltjes transform difference equation

$$
\begin{aligned}
& (t+1)(t+b+1) S(t+1)-z\left(t+a_{1}\right)\left(t+a_{2}\right) S(t) \\
& =\left[t+b+1-z\left(t+a_{1}+a_{2}\right)\right] \nu_{0}+(1-z) \nu_{1} .
\end{aligned}
$$




\subsubsection{Reduced-Uvarov Meixner polynomials}

Let $(\omega, \Omega) \in\{(0,0),(-a, a+1)\}$.

Special values

$$
a_{1} \rightarrow \Omega, \quad a_{2} \rightarrow a, \quad b \rightarrow \Omega-1 .
$$

Linear functional

$$
L_{U}[r]=\sum_{x=0}^{\infty} r(x)(a)_{x} \frac{z^{x}}{x !}+M r(\omega) .
$$

Pearson equation

$$
\frac{\varrho_{U}(x+1)}{\varrho_{U}(x)}=\frac{z(x+\Omega)(x+a)}{(x+\Omega)(x+1)} .
$$

Moments

$$
\nu_{n}^{U}(z)=z^{n}(a)_{n}(1-z)^{-a-n}+M \phi_{n}(\omega), \quad n \in \mathbb{N}_{0} .
$$

Stieltjes transform difference equation

$$
(t+\Omega)\left[(t+1) S_{U}(t+1)-z(t+a) S_{U}(t)\right]=[(1-z)(t+\Omega)-a z] \nu_{0}^{U}+(1-z) \nu_{1}^{U} .
$$

Stieltjes transform $M$-dependent difference equation

$$
(t+1) S_{U}(t+1)-z(t+a) S_{U}(t)=(1-z) \nu_{0}+M\left[\frac{t+1}{t-\omega+1}-\frac{z(t+a)}{t-\omega}\right] .
$$

\subsubsection{Christoffel Meixner polynomials}

Let $\omega \notin\left\{-a, 0, a z(1-z)^{-1}\right\}$.

Special values

$$
a_{1} \rightarrow a, \quad a_{2} \rightarrow-\omega+1, \quad b \rightarrow-\omega-1 .
$$

Linear functional

$$
L_{C}[r]=\sum_{x=0}^{\infty} r(x)(x-\omega)(a)_{x} \frac{z^{x}}{x !}
$$


Pearson equation

$$
\frac{\varrho_{C}(x+1)}{\varrho_{C}(x)}=\frac{z(x+a)(x+1-\omega)}{(x+1)(x-\omega)} .
$$

Moments

$\nu_{n}^{C}(z)=\nu_{n+1}+(n-\omega) \nu_{n}=z^{n}(a)_{n}(1-z)^{-a-n-1}(a z+\omega z+n-\omega), \quad n \in \mathbb{N}_{0}$.

Stieltjes transform difference equation

$$
\begin{aligned}
& (t-\omega)(t+1) S_{C}(t+1)-(t+1-\omega) z(t+a) S_{C}(t) \\
& =\nu_{0}\left[(a z-\omega+\omega z) t+\left(\omega^{2}+a z+\omega z-a z \omega-z \omega^{2}\right)\right] .
\end{aligned}
$$

Note that if we use (54), we have

$$
\nu_{0}^{C}=\frac{a z+\omega z-\omega}{1-z} \nu_{0}, \quad \nu_{1}^{C}=\frac{z a(a z+\omega z+1-\omega)}{(1-z)^{2}} \nu_{0},
$$

and therefore we can also write

$$
\begin{aligned}
& (t-\omega)(t+1) S_{C}(t+1)-(t+1-\omega) z(t+a) S_{C}(t) \\
& =[t-\omega-z(t+a-\omega+1)] \nu_{0}^{C}+(1-z) \nu_{1}^{C} .
\end{aligned}
$$

\subsubsection{Geronimus Meixner polynomials}

Let $\omega \notin\{-1,1-a\} \cup \mathbb{N}_{0}$.

Special values

$$
a_{1} \rightarrow a, \quad a_{2} \rightarrow-\omega, \quad b \rightarrow-\omega .
$$

Linear functional

$$
L_{G}[r]=\sum_{x=0}^{\infty} \frac{r(x)}{x-\omega}(a)_{x} \frac{z^{x}}{x !}+M r(\omega) .
$$

Pearson equation

$$
\frac{\varrho_{G}(x+1)}{\varrho_{G}(x)}=\frac{z(x+a)(x-\omega)}{(x+1)(x+1-\omega)} .
$$


Moments

$$
\nu_{n}^{G}(z)=\phi_{n}(\omega)\left[M-S(\omega)+(1-z)^{-a} \sum_{k=0}^{n-1} \frac{z^{k}(a)_{k}(1-z)^{-k}}{\phi_{k+1}(\omega)}\right], \quad n \in \mathbb{N}_{0} .
$$

Stieltjes transform difference equation

$$
\begin{aligned}
& (t+1)(t-\omega+1) S_{G}(t+1)-z(t+a)(t-\omega) S_{G}(t) \\
& =(1-z) \nu_{0}+\nu_{0}^{G}[(1-z) t+(1-a z)] .
\end{aligned}
$$

Note that if we use (34), we have

$$
\nu_{1}^{G}-\omega \nu_{0}^{G}=\nu_{0},
$$

and therefore we can also write

$$
\begin{aligned}
& (t+1)(t-\omega+1) S_{G}(t+1)-z(t+a)(t-\omega) S_{G}(t) \\
& =[(1-z) t+z \omega-\omega-a z+1] \nu_{0}^{G}+(1-z) \nu_{1}^{G} .
\end{aligned}
$$

\subsubsection{Truncated Meixner polynomials}

Special values

$$
a_{1} \rightarrow a, \quad a_{2} \rightarrow-N, \quad b \rightarrow-N-1 .
$$

Linear functional

$$
L_{T}[r]=\sum_{x=0}^{N} r(x)(a)_{x} \frac{z^{x}}{x !}
$$

Moments

$$
\nu_{n}^{T}=(a)_{N} \frac{z^{N}}{(N-n) !}{ }_{2} F_{1}\left[\begin{array}{c}
n-N, 1 \\
1-N-a
\end{array} ; \frac{1}{z}\right], \quad n \in \mathbb{N}_{0} .
$$

Pearson equation

$$
\frac{\varrho_{T}(x+1)}{\varrho_{T}(x)}=\frac{z(x+a)(x-N)}{(x-N)(x+1)} .
$$

Stieltjes transform difference equation

$$
(t-N)\left[(t+1) S_{T}(t+1)-z(t+a) S_{T}(t)\right]=[(t-N)(1-z)-a z] \nu_{0}^{T}+(1-z) \nu_{1}^{T} .
$$




\subsubsection{Symmetrized Generalized Krawtchouk polynomials}

Special values

$$
a_{1} \rightarrow a, \quad a_{2} \rightarrow-2 m, \quad b \rightarrow-2 m-a, \quad z \rightarrow-1, \quad x \rightarrow x+m .
$$

Linear functional

$$
L_{\varsigma}[r]=\varrho_{\varsigma}(0) \sum_{x=-m}^{m} r(x) \frac{(a+m)_{x}(-m)_{x}}{(-m-a+1)_{x}(m+1)_{x}}(-1)^{x},
$$

where

$$
\varrho_{\varsigma}(0)=(-1)^{m}\left(\begin{array}{c}
2 m \\
m
\end{array}\right) \frac{(a)_{m}}{(a+m)_{m}} .
$$

Pearson equation

$$
\frac{\varrho_{\varsigma}(x+1)}{\varrho_{\varsigma}(x)}=\frac{-(x+a+m)(x-m)}{(x+1-a-m)(x+1+m)} .
$$

Moments on the basis $\left\{\bar{\phi}_{n}(x)\right\}_{n \geq 0}$

$$
\nu_{n}^{\varsigma}=(-1)^{n} \frac{(a)_{n}(-2 m)_{n}}{(-2 m-a)_{n}}{ }_{2} F_{1}\left[\begin{array}{c}
-2 m+n, a+n \\
-2 m-a+n
\end{array} ;-1\right], \quad n \in \mathbb{N}_{0} .
$$

Stieltjes transform difference equation

$(t+m+1)(t-m-a+1) S_{\varsigma}(t+1)+(t-m)(t+m+a) S_{\varsigma}(t)=(2 t-2 m+1) \nu_{0}^{\varsigma}+2 \nu_{1}^{\varsigma}$.

\section{$5.5(3,2 ; N, 1)$ : Generalized Hahn polynomials of type II}

Linear functional

$$
L[r]=\sum_{x=0}^{N} r(x) \frac{\left(a_{1}\right)_{x}\left(a_{2}\right)_{x}(-N)_{x}}{\left(b_{1}+1\right)_{x}\left(b_{2}+1\right)_{x}} \frac{1}{x !} .
$$

Pearson equation

$$
\frac{\varrho(x+1)}{\varrho(x)}=\frac{\left(x+a_{1}\right)\left(x+a_{2}\right)(x-N)}{\left(x+b_{1}+1\right)\left(x+b_{2}+1\right)(x+1)} .
$$


Moments

$$
\nu_{n}=\frac{(-N)_{n}\left(a_{1}\right)_{n}\left(a_{2}\right)_{n}}{\left(b_{1}+1\right)_{n}\left(b_{2}+1\right)_{n}} F_{2}\left[\begin{array}{c}
-N+n, a_{1}+n, a_{2}+n \\
b_{1}+1+n, b_{2}+1+n
\end{array} ; 1\right], \quad n \in \mathbb{N}_{0} .
$$

Stieltjes transform difference equation

$$
\begin{aligned}
& \left(t+b_{1}+1\right)\left(t+b_{2}+1\right)(t+1) S(t+1)-\left(t+a_{1}\right)\left(t+a_{2}\right)(t-N) S(t) \\
& =\left[\left(N+2+b_{1}+b_{2}-a_{1}-a_{2}\right) t+b_{1}+b_{2}+b_{1} b_{2}-a_{1}-a_{2}-a_{1} a_{2}+N a_{1}+N a_{2}+1\right] \nu_{0} \\
& +\left(N+b_{1}+b_{2}-a_{1}-a_{2}+1\right) \nu_{1} .
\end{aligned}
$$

\subsubsection{Reduced-Uvarov Hahn polynomials}

Let $(\omega, \Omega) \in\{(0,0),(-a, a+1),(-b, b),(N,-N+1)\}$.

Special values

$$
a_{1} \rightarrow a, \quad a_{2} \rightarrow \Omega, \quad b_{1} \rightarrow \Omega-1, \quad b_{2} \rightarrow a .
$$

Linear functional

$$
L_{U}[r]=\sum_{x=0}^{N} r(x) \frac{(a)_{x}(-N)_{x}}{(b+1)_{x}} \frac{1}{x !}+M r(\omega) .
$$

Pearson equation

$$
\frac{\varrho_{U}(x+1)}{\varrho_{U}(x)}=\frac{(x+a)(x+\Omega)(x-N)}{(x+b+1)(x+\Omega)(x+1)} .
$$

Moments

$$
\nu_{n}^{U}=\frac{(-N)_{n}(a)_{n}}{(b+1)_{n}} \frac{(b+1-a)_{N-n}}{(b+1+n)_{N-n}}+M \phi_{n}(\omega), \quad n \in \mathbb{N}_{0} .
$$

Stieltjes transform difference equation

$$
\begin{gathered}
(t+\Omega)\left[(t+b+1)(t+1) S_{U}(t+1)-(t+a)(t-N)\right] S_{U}(t) \\
=[(N-a+b+1) t+(N-a+b+1) \Omega+a N] \nu_{0}^{U}+(N+b-a) \nu_{1}^{U} .
\end{gathered}
$$

Stieltjes transform $M$-dependent difference equation

$$
\begin{aligned}
& (t+b+1)(t+1) S_{U}(t+1)-(t-N)(t+a) S_{U}(t) \\
& =(N+b-a+1) \nu_{0}+M\left[\frac{(t+b+1)(t+1)}{t-\omega+1}-\frac{(t-N)(t+a)}{t-\omega}\right] .
\end{aligned}
$$




\subsubsection{Christoffel Hahn polynomials}

Let $\omega \notin\left\{-a,-b, 0, N,-\frac{a N}{b-a+N}\right\}$.

Special values

$$
a_{1} \rightarrow a, \quad a_{2} \rightarrow-\omega+1, \quad b_{1} \rightarrow b, \quad b_{2} \rightarrow-\omega-1 .
$$

Linear functional

$$
L_{C}[r]=\sum_{x=0}^{\infty} r(x)(x-\omega) \frac{(a)_{x}(-N)_{x}}{(b+1)_{x}} \frac{1}{x !} .
$$

Pearson equation

$$
\frac{\varrho_{C}(x+1)}{\varrho_{C}(x)}=\frac{(x+a)(x-N)(x+1-\omega)}{(x+b+1)(x+1)(x-\omega)} .
$$

Moments

$$
\nu_{n}^{C}(z)=\nu_{n+1}+(n-\omega) \nu_{n}=\left[n-\omega-\frac{(a+n)(N-n)}{b-a+N-n}\right] \nu_{n}, \quad n \in \mathbb{N}_{0} .
$$

Stieltjes transform difference equation

$$
\begin{aligned}
& (t-\omega)(t+b+1)(t+1) S_{C}(t+1)-(t-\omega+1)(t-N)(t+a) S_{C}(t) \\
& =\left[(a \omega-N \omega-b \omega-N a) t+a \omega-N \omega+\omega^{2}+N \omega^{2}-a \omega^{2}+b \omega^{2}-N a+N a \omega\right] \nu_{0} .
\end{aligned}
$$

Note that if we use (55), we have

$\nu_{0}^{C}=-\frac{(a+\omega) N+(b-a) \omega}{b-a+N} \nu_{0}, \quad \nu_{1}^{C}=\frac{(N a+N \omega-a \omega+b \omega-b-\omega) N a}{(b-a+N)(b-a+N-1)} \nu_{0}$,

and therefore we can also write

$$
\begin{aligned}
& (t-\omega)(t+b+1)(t+1) S_{C}(t+1)-(t-\omega+1)(t-N)(t+a) S_{C}(t) \\
= & {[(N-a+b) t+N a-N \omega+a \omega-b \omega+N-a-\omega] \nu_{0}^{C}+(N-a+b-1) \nu_{1}^{C} . }
\end{aligned}
$$




\subsubsection{Geronimus Hahn polynomials}

Let $\omega \notin\{-b-1,-1,1-a, N+1\} \cup \mathbb{N}_{0}$.

Special values

$$
a_{1} \rightarrow a, \quad a_{2} \rightarrow-\omega, \quad b \rightarrow-\omega .
$$

Linear functional

$$
L_{G}[r]=\sum_{x=0}^{\infty} \frac{r(x)}{x-\omega} \frac{(a)_{x}(-N)_{x}}{(b+1)_{x}} \frac{1}{x !}+M r(\omega) .
$$

Pearson equation

$$
\frac{\varrho_{G}(x+1)}{\varrho_{G}(x)}=\frac{(x+a)(x-N)(x-\omega)}{(x+b+1)(x+1)(x+1-\omega)} .
$$

Moments

$$
\nu_{n}^{G}(z)=\phi_{n}(\omega)\left[M-S(\omega)+\sum_{k=0}^{n-1} \frac{\nu_{k}}{\phi_{k+1}(\omega)}\right], \quad n \in \mathbb{N}_{0} .
$$

Stieltjes transform difference equation

$$
\begin{gathered}
\xi_{G}(t)=\xi(t)+[\sigma(t+1)-\eta(t)] \nu_{0}^{G}, \\
(t-\omega+1)(t+b+1)(t+1) S_{G}(t+1)-(t-\omega)(t-N)(t+a) S_{G}(t) \\
=(b+1-a+N) \nu_{0}+[(N-a+b+2) t+b+N a+1] \nu_{0}^{G} .
\end{gathered}
$$

Note that if we use (34), we have

$$
\nu_{1}^{G}-\omega \nu_{0}^{G}=\nu_{0},
$$

and therefore we can also write

$$
\begin{aligned}
& (t-\omega+1)(t+b+1)(t+1) S_{G}(t+1)-(t-\omega)(t-N)(t+a) S_{G}(t) \\
& =[(N-a+b+2) t+N a-N \omega+a \omega-b \omega+b-\omega+1] \nu_{0}^{G}+(b-1-a+N) \nu_{1}^{G} .
\end{aligned}
$$




\subsubsection{Symmetrized Hahn polynomials}

Special values

$a_{1} \rightarrow a, \quad a_{2} \rightarrow-N-b, \quad b_{1} \rightarrow b, \quad b_{2} \rightarrow-N-a, \quad N \rightarrow 2 m, \quad x \rightarrow x+m$.

Linear functional

$$
L_{\varsigma}[r]=\varrho_{\varsigma}(0) \sum_{x=-m}^{m} r(x) \frac{(m+a)_{x}(-m-b)_{x}(-m)_{x}}{(1-m-a)_{x}(m+b+1)_{x}(m+1)_{x}},
$$

where

$$
\varrho_{\varsigma}(0)=(-1)^{m}\left(\begin{array}{c}
2 m \\
m
\end{array}\right) \frac{(a)_{m}}{(a+m)_{m}} \frac{(b+1+m)_{m}}{(b+1)_{m}} .
$$

Pearson equation

$$
\frac{\varrho_{\varsigma}(x+1)}{\varrho_{\varsigma}(x)}=\frac{(x+m+a)(x-m-b)(x-m)}{(x+1-m-a)(x+1+m+b)(x+1+m)} .
$$

Moments on the basis $\left\{\bar{\phi}_{n}(x)\right\}_{n \geq 0}$

$$
\nu_{n}^{\varsigma}=\frac{(-2 m)_{n}(a)_{n}(-2 m-b)_{n}}{(b+1)_{n}(-2 m-a+1)_{n}}{ }_{3} F_{2}\left[\begin{array}{c}
-2 m+n, a+n,-2 m-b+n \\
b+1+n,-2 m-a+1+n
\end{array} ; 1\right], \quad n \in \mathbb{N}_{0} .
$$

Stieltjes transform difference equation

$$
\begin{aligned}
& (t+1-m-a)(t+1+m+b)(t+1+m) S_{\varsigma}(t+1)-(t+m+a)(t-m-b)(t-m) S_{\varsigma}(t) \\
& =\left[2(1+b-a+m) t+2 a m-2 b m-2 m^{2}-a+b+1\right] \nu_{0}^{\varsigma}+(1+2 m-2 a+2 b) \nu_{1}^{\varsigma} .
\end{aligned}
$$

\subsubsection{Symmetrized polynomials of type $(3,0 ; N)$}

For a definition of the polynomials of type $(3,0 ; N)$, see Section 6.4.

Special values

$$
b_{1} \rightarrow-N-a_{1}, \quad b_{2} \rightarrow-N-a_{2}, \quad N \rightarrow 2 m, \quad x \rightarrow x+m .
$$

Linear functional

$$
L_{\varsigma}[r]=\varrho_{\varsigma}(0) \sum_{x=-m}^{m} r(x) \frac{\left(a_{1}+m\right)_{x}\left(a_{2}+m\right)_{x}(-m)_{x}}{\left(1-a_{1}-m\right)_{x}\left(1-a_{2}-m\right)_{x}(m+1)_{x}},
$$


where

$$
\varrho_{\varsigma}(0)=(-1)^{m}\left(\begin{array}{c}
2 m \\
m
\end{array}\right) \frac{\left(a_{1}\right)_{m}}{\left(a_{1}+m\right)_{m}} \frac{\left(a_{2}\right)_{m}}{\left(a_{2}+m\right)_{m}} .
$$

Pearson equation

$$
\frac{\varrho_{\varsigma}(x+1)}{\varrho_{\varsigma}(x)}=\frac{\left(x+a_{1}+m\right)\left(x+a_{2}+m\right)(x-m)}{\left(x+1-a_{1}-m\right)\left(x+1-a_{2}-m\right)(x+1+m)} .
$$

Moments on the basis $\left\{\bar{\phi}_{n}(x)\right\}_{n \geq 0}$

$\nu_{n}^{\varsigma}=\frac{(-2 m)_{n}\left(a_{1}\right)_{n}\left(a_{2}\right)_{n}}{\left(1-a_{1}-2 m\right)_{n}\left(1-a_{2}-2 m\right)_{n}}{ }_{3} F_{2}\left[\begin{array}{c}-2 m+n, a_{1}+n, a_{2}+n \\ 1-a_{1}-2 m+n, 1-a_{2}-2 m+n\end{array} ; 1\right], \quad n \in \mathbb{N}_{0}$.

Stieltjes transform difference equation

$$
\begin{aligned}
& \left(t+1-a_{1}-m\right)\left(t+1-a_{2}-m\right)(t+1+m) S_{\varsigma}(t+1) \\
& -\left(t+a_{1}+m\right)\left(t+a_{2}+m\right)(t-m) S_{\varsigma}(t) \\
& =\left[-2\left(m+a_{1}+a_{2}-1\right) t+2 m^{2}-2 m+1+(2 m-1)\left(a_{1}+a_{2}\right)\right] \nu_{0}^{\varsigma} \\
& +\left(-2 m+1-2 a_{1}-2 a_{2}\right) \nu_{1}^{\varsigma} .
\end{aligned}
$$

\section{Semiclassical polynomials of class 2}

In this section, we consider all families of polynomials of class 2 . We have 7 main cases, corresponding to

$$
(p, q)=(0,2),(1,2),(2,2),(3,0),(3,1),(3,2),(4,3) .
$$

There are also 25 subcases.

\subsection{Polynomials of type $(0,2)$}

Linear functional

$$
L[r]=\sum_{x=0}^{\infty} r(x) \frac{1}{\left(b_{1}+1\right)_{x}\left(b_{2}+1\right)_{x}} \frac{z^{x}}{x !} .
$$

Pearson equation

$$
\frac{\varrho(x+1)}{\varrho(x)}=\frac{z}{\left(x+b_{1}+1\right)\left(x+b_{2}+1\right)(x+1)} .
$$


Moments

$\nu_{n}(z)=\frac{z^{n}}{\left(b_{1}+1\right)_{n}\left(b_{2}+1\right)_{n}}{ }_{0} F_{2}\left[\begin{array}{c}- \\ b_{1}+1+n, b_{2}+1+n\end{array} ; z\right], \quad n \in \mathbb{N}_{0}$.

Stieltjes transform difference equation

$$
\begin{aligned}
& \left(t+b_{1}+1\right)\left(t+b_{2}+1\right)(t+1) S(t+1)-z S(t) \\
& =\left(t+b_{1}+1\right)\left(t+b_{2}+1\right) \nu_{0}+\left(t+b_{1}+b_{2}+2\right) \nu_{1}+\nu_{2}
\end{aligned}
$$

\subsection{Polynomials of type $(1,2)$}

Linear functional

$$
L[r]=\sum_{x=0}^{\infty} r(x) \frac{(a)_{x}}{\left(b_{1}+1\right)_{x}\left(b_{2}+1\right)_{x}} \frac{z^{x}}{x !} .
$$

Pearson equation

$$
\frac{\varrho(x+1)}{\varrho(x)}=\frac{z(x+a)}{\left(x+b_{1}+1\right)\left(x+b_{2}+1\right)(x+1)} .
$$

Moments

$$
\nu_{n}(z)=z^{n} \frac{(a)_{n}}{\left(b_{1}+1\right)_{n}\left(b_{2}+1\right)_{n}}{ }_{1} F_{2}\left[\begin{array}{c}
a+n \\
b_{1}+1+n, b_{2}+1+n
\end{array} ; z\right], \quad n \in \mathbb{N}_{0} .
$$

Stieltjes transform difference equation

$$
\begin{aligned}
& \left(t+b_{1}+1\right)\left(t+b_{2}+1\right)(t+1) S(t+1)-z(t+a) S(t) \\
& =\left[\left(t+b_{1}+1\right)\left(t+b_{2}+1\right)-z\right] \nu_{0}+\left(t+b_{1}+b_{2}+2\right) \nu_{1}+\nu_{2}
\end{aligned}
$$

\subsubsection{Reduced-Uvarov Generalized Charlier polynomials}

Let $\omega \in\{0,-b\}$.

Special values

$$
a \rightarrow-\omega, \quad b_{1} \rightarrow b, \quad b_{2} \rightarrow-\omega-1 .
$$


Linear functional

$$
L_{U}[r]=\sum_{x=0}^{\infty} r(x) \frac{1}{(b+1)_{x}} \frac{z^{x}}{x !}+M r(\omega) .
$$

Pearson equation

$$
\frac{\varrho_{U}(x+1)}{\varrho_{U}(x)}=\frac{z(x-\omega)}{(x+b+1)(x-\omega)(x+1)} .
$$

Moments

$$
\nu_{n}^{U}(z)=\frac{z^{n}}{(b+1)_{n}}{ }_{0} F_{1}\left[\begin{array}{c}
- \\
b+1+n
\end{array} ; z\right]+M \phi_{n}(\omega), \quad n \in \mathbb{N}_{0} .
$$

Stieltjes transform difference equation

$$
\begin{aligned}
& (t-\omega)\left[(t+b+1)(t+1) S_{U}(t+1)-z S_{U}(t)\right] \\
& =[(t+b+1)(t+b)-z] \nu_{0}^{U}+(t+2 b+1) \nu_{1}^{U}+\nu_{2}^{U} .
\end{aligned}
$$

Stieltjes transform $M$-dependent difference equation

$$
(t+b+1)(t+1) S_{U}(t+1)-z S_{U}(t)=(t+b+1) \nu_{0}+\nu_{1}+M\left[\frac{(t+b+1)(t+1)}{t-\omega+1}-\frac{z}{t-\omega}\right] .
$$

\subsubsection{Christoffel generalized Charlier polynomials}

Let $\omega \notin\left\{-b, 0, \frac{\nu_{1}}{\nu_{0}}\right\}$.

Special values

$$
a \rightarrow-\omega+1, \quad b_{1} \rightarrow b, \quad b_{2} \rightarrow-\omega-1 .
$$

Linear functional

$$
L_{C}[r]=\sum_{x=0}^{\infty} r(x)(x-\omega) \frac{1}{(b+1)_{x}} \frac{z^{x}}{x !} .
$$

Pearson equation

$$
\frac{\varrho_{C}(x+1)}{\varrho_{C}(x)}=\frac{z(x+1-\omega)}{(x+b+1)(x+1)(x-\omega)} .
$$


Moments

$$
\nu_{n}^{C}(z)=\nu_{n+1}+(n-\omega) \nu_{n}, \quad n \in \mathbb{N}_{0} .
$$

Stieltjes transform difference equation

$$
\begin{aligned}
& (t-\omega)(t+b+1)(t+1) S_{C}(t+1)-z(t-\omega+1) S_{C}(t) \\
& =\left[-\omega t^{2}+\left(z-\omega-b \omega+\omega^{2}\right) t+\left(z-z \omega+\omega^{2}+b \omega^{2}\right)\right] \nu_{0}+(t-\omega)(t-\omega+1) \nu_{1} .
\end{aligned}
$$

\subsubsection{Geronimus generalized Charlier polynomials}

Let $\omega \notin\{-b-1,-1\} \cup \mathbb{N}_{0}$.

Special values

$$
a \rightarrow-\omega, \quad b_{1} \rightarrow b, \quad b_{2} \rightarrow-\omega .
$$

Linear functional

$$
L_{G}[r]=\sum_{x=0}^{\infty} \frac{r(x)}{x-\omega} \frac{1}{(b+1)_{x}} \frac{z^{x}}{x !}+M r(\omega) .
$$

Pearson equation

$$
\frac{\varrho_{G}(x+1)}{\varrho_{G}(x)}=\frac{z(x-\omega)}{(x+b+1)(x+1)(x+1-\omega)} .
$$

Moments

$$
\nu_{n}^{G}(z)=\phi_{n}(\omega)\left[M-S(\omega)+\sum_{k=0}^{n-1} \frac{\nu_{k}}{\phi_{k+1}(\omega)}\right], \quad n \in \mathbb{N}_{0} .
$$

Stieltjes transform difference equation

$$
\begin{aligned}
& (t-\omega+1)(t+b+1)(t+1) S_{G}(t+1)-(t-\omega) z S_{G}(t) \\
& =(t+b+1) \nu_{0}+\nu_{1}+[(t+b+1)(t+1)-z] \nu_{0}^{G} .
\end{aligned}
$$

\subsubsection{Truncated generalized Charlier polynomials}

Special values

$$
a \rightarrow-N, \quad b_{1} \rightarrow b, \quad b_{2} \rightarrow-N-1 .
$$

Linear functional

$$
L_{T}[r]=\sum_{x=0}^{N} r(x) \frac{1}{(b+1)_{x}} \frac{z^{x}}{x !}
$$


Pearson equation

$$
\frac{\varrho_{T}(x+1)}{\varrho_{T}(x)}=\frac{z(x-N)}{(x+b+1)(x-N)(x+1)} .
$$

Moments

$$
\nu_{n}^{T}(z)=\frac{1}{(b+1)_{N}} \frac{z^{N}}{(N-n) !}{ }_{3} F_{0}\left[\begin{array}{c}
n-N, 1,-N-b \\
-
\end{array} ; \frac{1}{z}\right], \quad n \in \mathbb{N}_{0} .
$$

Stieltjes transform difference equation

$$
\begin{aligned}
& (t-N)\left[(t+b+1)(t+1) S_{T}(t+1)-z S_{T}(t)\right] \\
& =[(t+b+1)(t-N)-z] \nu_{0}^{T}+(t+b+1-N) \nu_{1}^{T}+\nu_{2}^{T} .
\end{aligned}
$$

\subsection{Polynomials of type $(2,2)$}

Linear functional

$$
L[r]=\sum_{x=0}^{\infty} r(x) \frac{\left(a_{1}\right)_{x}\left(a_{2}\right)_{x}}{\left(b_{1}+1\right)_{x}\left(b_{2}+1\right)_{x}} \frac{z^{x}}{x !}
$$

Pearson equation

$$
\frac{\varrho(x+1)}{\varrho(x)}=\frac{z\left(x+a_{1}\right)\left(x+a_{2}\right)}{\left(x+b_{1}+1\right)\left(x+b_{2}+1\right)(x+1)} .
$$

Moments

$$
\nu_{n}(z)=z^{n} \frac{\left(a_{1}\right)_{n}\left(a_{2}\right)_{n}}{\left(b_{1}+1\right)_{n}\left(b_{2}+1\right)_{n}}{ }_{2} F_{2}\left[\begin{array}{c}
a_{1}+n, a_{2}+n \\
b_{1}+1+n, b_{2}+1+n
\end{array} ; z\right], \quad n \in \mathbb{N}_{0} .
$$

Stieltjes transform difference equation

$$
\begin{aligned}
& \left(t+b_{1}+1\right)\left(t+b_{2}+1\right)(t+1) S(t+1)-z\left(t+a_{1}\right)\left(t+a_{2}\right) S(t) \\
& =\left[\left(t+b_{1}+1\right)\left(t+b_{2}+1\right)-\left(t+a_{1}+a_{2}\right) z\right] \nu_{0}+\left(t+b_{1}+b_{2}+2-z\right) \nu_{1}+\nu_{2} .
\end{aligned}
$$




\subsubsection{Uvarov Charlier polynomials}

Let $\omega \neq 0$.

Special values

$$
a_{1} \rightarrow-\omega, \quad a_{2} \rightarrow-\omega+1, \quad b_{1} \rightarrow-\omega-1, \quad b_{2} \rightarrow-\omega .
$$

Linear functional

$$
L_{U}[r]=\sum_{x=0}^{\infty} r(x) \frac{z^{x}}{x !}+M r(\omega) .
$$

Pearson equation

$$
\frac{\varrho_{U}(x+1)}{\varrho_{U}(x)}=\frac{z(x-\omega)(x-\omega+1)}{(x-\omega)(x-\omega+1)(x+1)} .
$$

Moments

$$
\nu_{n}^{U}(z)=z^{n} e^{z}+M \phi_{n}(\omega), \quad n \in \mathbb{N}_{0} .
$$

Stieltjes transform difference equation

$$
\begin{aligned}
& (t-\omega)(t-\omega+1)\left[(t+1) S_{U}(t+1)-z S_{U}(t)\right] \\
& =[(t-\omega)(t-\omega+1)-(t-2 \omega+1) z] \nu_{0}^{U}+(t-z-2 \omega+1) \nu_{1}^{U}+\nu_{2}^{U} .
\end{aligned}
$$

Stieltjes transform $M$-dependent difference equation

$$
(t+1) S_{U}(t+1)-z S_{U}(t)=\nu_{0}+M\left(\frac{t+1}{t+1-\omega}-\frac{z}{t-\omega}\right) .
$$

\subsubsection{Reduced-Uvarov generalized Meixner polynomials}

Let $(\omega, \Omega) \in\{(-a, a+1),(-b, b),(0,0)\}$.

Special values

$$
a_{1} \rightarrow a, \quad a_{2} \rightarrow \Omega, \quad b_{1} \rightarrow b, \quad b_{2} \rightarrow \Omega-1 .
$$

Linear functional

$$
L_{U}[r]=\sum_{x=0}^{\infty} r(x) \frac{(a)_{x}}{(b+1)_{x}} \frac{z^{x}}{x !}+M r(\omega) .
$$


Pearson equation

$$
\frac{\varrho_{U}(x+1)}{\varrho_{U}(x)}=\frac{z(x+a)(x+\Omega)}{(x+b+1)(x+\Omega)(x+1)} .
$$

Moments

$$
\nu_{n}^{U}(z)=z^{n} \frac{(a)_{n}}{(b+1)_{n}}{ }_{1} F_{1}\left[\begin{array}{c}
a+n \\
b+1+n
\end{array} ; z\right]+M \phi_{n}(\omega), \quad n \in \mathbb{N}_{0} .
$$

Stieltjes transform difference equation

$$
\begin{aligned}
& (t+\Omega)\left[(t+b+1)(t+1) S_{U}(t+1)-z(t+a) S_{U}(t)\right] \\
& =[(t+b+1)(t+\Omega)-(t+a+\Omega) z] \nu_{0}^{U}+(t+b+1+\Omega-z) \nu_{1}^{U}+\nu_{2}^{U} .
\end{aligned}
$$

Stieltjes transform $M$-dependent difference equation

$$
\begin{aligned}
& (t+b+1)(t+1) S_{U}(t+1)-z(t+a) S_{U}(t) \\
& =(t+b+1-z) \nu_{0}+\nu_{1}+M\left[\frac{(t+b+1)(t+1)}{t+1-\omega}-\frac{z(t+a)}{t-\omega}\right] .
\end{aligned}
$$

\subsubsection{Christoffel generalized Meixner polynomials}

Let $\omega \notin\left\{-a,-b, 0, \frac{\nu_{1}}{\nu_{0}}\right\}$.

Special values

$$
a_{1} \rightarrow a, \quad a_{2} \rightarrow-\omega+1, \quad b_{1} \rightarrow b, \quad b_{2} \rightarrow-\omega-1 .
$$

Linear functional

$$
L_{C}[r]=\sum_{x=0}^{\infty} r(x)(x-\omega) \frac{(a)_{x}}{(b+1)_{x}} \frac{z^{x}}{x !} .
$$

Pearson equation

$$
\frac{\varrho_{C}(x+1)}{\varrho_{C}(x)}=\frac{z(x+a)(x+1-\omega)}{(x+1)(x+b+1)(x-\omega)} .
$$

Moments

$$
\nu_{n}^{C}(z)=\nu_{n+1}+(n-\omega) \nu_{n}, \quad n \in \mathbb{N}_{0} .
$$


Stieltjes transform difference equation

$$
\begin{aligned}
& (t-\omega)(t+b+1)(t+1) S_{C}(t+1)-z(t-\omega+1)(t+a) S_{C}(t) \\
& =\left[-\omega t^{2}+\left(z \omega-b \omega-\omega+\omega^{2}+a z\right) t+z \omega+\omega^{2}+b \omega^{2}-z \omega^{2}+a z-a z \omega\right] \nu_{0} \\
& +(t-\omega)(t-\omega+1) \nu_{1} .
\end{aligned}
$$

\subsubsection{Geronimus generalized Meixner polynomials}

Let $\omega \notin\{-b-1,-1,1-a\} \cup \mathbb{N}_{0}$.

Special values

$$
a_{1} \rightarrow a, \quad a_{2} \rightarrow-\omega, \quad b_{1} \rightarrow b, \quad b_{2} \rightarrow-\omega
$$

Linear functional

$$
L_{G}[r]=\sum_{x=0}^{\infty} \frac{r(x)}{x-\omega} \frac{(a)_{x}}{(b+1)_{x}} \frac{z^{x}}{x !}+M r(\omega) .
$$

Pearson equation

$$
\frac{\varrho_{G}(x+1)}{\varrho_{G}(x)}=\frac{z(x+a)(x-\omega)}{(x+1)(x+b+1)(x+1-\omega)} .
$$

Moments

$$
\nu_{n}^{G}(z)=\phi_{n}(\omega)\left[M-S(\omega)+\sum_{k=0}^{n-1} \frac{\nu_{k}}{\phi_{k+1}(\omega)}\right], \quad n \in \mathbb{N}_{0} .
$$

Stieltjes transform difference equation

$$
\begin{aligned}
& (t-\omega+1)(t+b+1)(t+1) S_{G}(t+1)-z(t-\omega)(t+a) S_{G}(t) \\
& =(t+b+1-z) \nu_{0}+\nu_{1}+[(t+b+1)(t+1)-z(t+a)] \nu_{0}^{G} .
\end{aligned}
$$

\subsubsection{Truncated generalized Meixner polynomials}

Special values

$$
a_{1} \rightarrow a, \quad a_{2} \rightarrow-N, \quad b_{1} \rightarrow b, \quad b_{2} \rightarrow-N-1 .
$$


Linear functional

$$
L_{T}[r]=\sum_{x=0}^{N} r(x) \frac{(a)_{x}}{(b+1)_{x}} \frac{z^{x}}{x !}
$$

Pearson equation

$$
\frac{\varrho_{T}(x+1)}{\varrho_{T}(x)}=\frac{z(x+a)(x-N)}{(x+b+1)(x-N)(x+1)} .
$$

Moments

$$
\nu_{n}^{T}(z)=\frac{(a)_{N}}{(b+1)_{N}} \frac{z^{N}}{(N-n) !}{ }_{3} F_{1}\left[\begin{array}{c}
n-N, 1,-N-b \\
1-N-a
\end{array} ; \frac{1}{z}\right], \quad n \in \mathbb{N}_{0} .
$$

Stieltjes transform difference equation

$$
\begin{aligned}
& (t-N)\left[(t+b+1)(t+1) S_{T}(t+1)-z(t+a) S_{T}(t)\right] \\
& =[(t+b+1)(t-N)-(t+a-N) z] \nu_{0}^{T}+(t+b-N+1-z) \nu_{1}^{T}+\nu_{2}^{T} .
\end{aligned}
$$

\subsection{Polynomials of type $(3,0 ; \mathrm{N})$}

Linear functional

$$
L[r]=\sum_{x=0}^{N} r(x)\left(a_{1}\right)_{x}\left(a_{2}\right)_{x}(-N)_{x} \frac{z^{x}}{x !} .
$$

Pearson equation

$$
\frac{\varrho(x+1)}{\varrho(x)}=\frac{z\left(x+a_{1}\right)\left(x+a_{2}\right)(x-N)}{x+1} .
$$

Moments

$$
\nu_{n}(z)=z^{n}(-N)_{n}\left(a_{1}\right)_{n}\left(a_{2}\right)_{n}{ }_{3} F_{0}\left[\begin{array}{c}
-N+n, a_{1}+n, a_{2}+n \\
-
\end{array} ; z\right], \quad n \in \mathbb{N}_{0} .
$$

Stieltjes transform difference equation

$$
\begin{aligned}
& (t+1) S(t+1)-z\left(t+a_{1}\right)\left(t+a_{2}\right)(t-N) S(t) \\
& =\left[-z t^{2}-\left(a_{1}+a_{2}-N\right) z t+z\left(N a_{1}+N a_{2}-a_{1} a_{2}\right)+1\right] \nu_{0} \\
& -\left(t+a_{1}+a_{2}+1-N\right) z \nu_{1}-z \nu_{2} .
\end{aligned}
$$




\subsection{Polynomials of type $(3,1 ; \mathrm{N})$}

Linear functional

$$
L[r]=\sum_{x=0}^{N} r(x) \frac{\left(a_{1}\right)_{x}\left(a_{2}\right)_{x}(-N)_{x}}{(b+1)_{x}} \frac{z^{x}}{x !}
$$

Pearson equation

$$
\frac{\varrho(x+1)}{\varrho(x)}=\frac{z\left(x+a_{1}\right)\left(x+a_{2}\right)(x-N)}{(x+b+1)(x+1)} .
$$

Moments

$$
\nu_{n}(z)=z^{n} \frac{(-N)_{n}\left(a_{1}\right)_{n}\left(a_{2}\right)_{n}}{(b+1)_{n}}{ }_{3} F_{1}\left[\begin{array}{c}
-N+n, a_{1}+n, a_{2}+n \\
b+1+n
\end{array} ; z\right], \quad n \in \mathbb{N}_{0} .
$$

Stieltjes transform difference equation

$$
\begin{aligned}
& (x+b+1)(t+1) S(t+1)-z\left(t+a_{1}\right)\left(t+a_{2}\right)(t-N) S(t) \\
& =\left[-z t^{2}+t-\left(a_{1}+a_{2}-N\right) z t+\left(N a_{1}+N a_{2}-a_{1} a_{2}\right) z+b+1\right] \nu_{0} \\
& +\left[1-\left(t+a_{1}+a_{2}+1-N\right) z\right] \nu_{1}-z \nu_{2} .
\end{aligned}
$$

\subsubsection{Reduced-Uvarov generalized Krawtchouk polynomials}

Let $(\omega, \Omega) \in\{(-a, a+1),(0,0),(N,-N+1)\}$.

Special values

$$
a_{1} \rightarrow a, \quad a_{2} \rightarrow \Omega, \quad b \rightarrow \Omega-1 .
$$

Linear functional

$$
L_{U}[r]=\sum_{x=0}^{N} r(x)(a)_{x}(-N)_{x} \frac{z^{x}}{x !}+M r(\omega) .
$$

Pearson equation

$$
\frac{\varrho_{U}(x+1)}{\varrho_{U}(x)}=\frac{z(x+a)(x+\Omega)(x-N)}{(x+\Omega)(x+1)} .
$$


Moments

$\nu_{n}^{U}(z)=z^{n}(-N)_{n}(a)_{n}{ }_{2} F_{0}\left[\begin{array}{c}-N+n, a+n \\ -\end{array} ; z+M \phi_{n}(\omega), \quad n \in \mathbb{N}_{0}\right.$.

Stieltjes transform difference equation

$$
\begin{aligned}
& (t+\Omega)\left[(t+1) S_{U}(t+1)-z(t+a)(t-N) S_{U}(t)\right] \\
& =\left[-z t^{2}+t-(a+\Omega-N) z t+(N a+N \Omega-a \Omega) z+\Omega\right] \nu_{0}^{U} \\
& +[1-(t+a+\Omega+1-N) z] \nu_{1}^{U}-z \nu_{2}^{U} .
\end{aligned}
$$

Stieltjes transform $M$-dependent difference equation

$$
\begin{aligned}
& (t+1) S_{U}(t+1)-z(t+a)(t-N) S_{U}(t) \\
& =[1-(t+a-N) z] \nu_{0}-z \nu_{1}+M\left[\frac{t+1}{t-\omega+1}-\frac{z(t+a)(t-N)}{t-\omega}\right] .
\end{aligned}
$$

\subsubsection{Christoffel generalized Krawtchouk polynomials}

Let $\omega \notin\left\{-a, 0, N, \frac{\nu_{1}}{\nu_{0}}\right\}$.

Special values

$$
a_{1} \rightarrow a, \quad a_{2} \rightarrow-\omega+1, \quad b \rightarrow-\omega-1 .
$$

Linear functional

$$
L_{C}[r]=\sum_{x=0}^{\infty} r(x)(x-\omega)(a)_{x}(-N)_{x} \frac{z^{x}}{x !} .
$$

Pearson equation

$$
\frac{\varrho_{C}(x+1)}{\varrho_{C}(x)}=\frac{z(x+a)(x-N)(x+1-\omega)}{(x+1)(x-\omega)} .
$$

Moments

$$
\nu_{n}^{C}(z)=\nu_{n+1}+(n-\omega) \nu_{n}, \quad n \in \mathbb{N}_{0} .
$$

Stieltjes transform difference equation

$$
\begin{aligned}
& (t-\omega)(t+1) S_{C}(t+1)-z(t-\omega+1)(t+a)(t-N) S_{C}(t) \\
& =\left[z \omega t^{2}-\left(\omega-z \omega+z \omega^{2}+N z \omega-a z \omega+N a z\right) t\right] \nu_{0} \\
& +\left(\omega^{2}-N z \omega+a z \omega+N z \omega^{2}-a z \omega^{2}-N a z+N a z \omega\right) \nu_{0}-z(t-\omega)(t-\omega+1) \nu_{1} .
\end{aligned}
$$




\subsubsection{Geronimus generalized Krawtchouk polynomials}

Let $\omega \notin\{-1,1-a, 1+N\} \cup \mathbb{N}_{0}$.

Special values

$$
a_{1} \rightarrow a, \quad a_{2} \rightarrow-\omega, \quad b \rightarrow-\omega .
$$

Linear functional

$$
L_{G}[r]=\sum_{x=0}^{\infty} \frac{r(x)}{x-\omega}(a)_{x}(-N)_{x} \frac{z^{x}}{x !}+M r(\omega) .
$$

Pearson equation

$$
\frac{\varrho_{G}(x+1)}{\varrho_{G}(x)}=\frac{z(x+a)(x-N)(x-\omega)}{(x+1)(x+1-\omega)} .
$$

Moments

$$
\nu_{n}^{G}(z)=\phi_{n}(\omega)\left[M-S(\omega)+\sum_{k=0}^{n-1} \frac{\nu_{k}}{\phi_{k+1}(\omega)}\right], \quad n \in \mathbb{N}_{0} .
$$

Stieltjes transform difference equation

$$
\begin{aligned}
& (t-\omega+1)(t+1) S_{G}(t+1)-z(t-\omega)(t+a)(t-N) S_{G}(t) \\
& =(-z t+N z-a z+1) \nu_{0}-z \nu_{1}+[t+1-z(t+a)(t-N)] \nu_{0}^{G} .
\end{aligned}
$$

\subsection{Polynomials of type $(3,2)$}

Linear functional

$$
L[r]=\sum_{x=0}^{\infty} r(x) \frac{\left(a_{1}\right)_{x}\left(a_{2}\right)_{x}\left(a_{3}\right)_{x}}{\left(b_{1}+1\right)_{x}\left(b_{2}+1\right)_{x}} \frac{z^{x}}{x !} .
$$

Pearson equation

$$
\frac{\varrho(x+1)}{\varrho(x)}=\frac{z\left(x+a_{1}\right)\left(x+a_{2}\right)\left(x+a_{3}\right)}{\left(x+b_{1}+1\right)\left(x+b_{2}+1\right)(x+1)} .
$$

Moments 


$$
\nu_{n}(z)=z^{n} \frac{\left(a_{1}\right)_{n}\left(a_{2}\right)_{n}\left(a_{3}\right)_{n}}{\left(b_{1}+1\right)_{n}\left(b_{2}+1\right)_{n}}{ }_{3} F_{2}\left[\begin{array}{c}
a_{1}+n, a_{2}+n, a_{3}+n \\
b_{1}+1+n, b_{2}+1+n
\end{array} ; z\right], \quad n \in \mathbb{N}_{0},
$$

where we choose the principal branch $z \in \mathbb{C} \backslash[1, \infty)$. Note that since

$b_{1}+1+n+b_{2}+1+n-\left(a_{1}+n+a_{2}+n+a_{3}+n\right) \leq 0, \quad n \geq b_{1}+b_{2}-a_{2}-a_{3}-a_{1}+2$,

$\nu_{n}(1)$ is undefined for $n \geq b_{1}+b_{2}-a_{2}-a_{3}-a_{1}+2$.

Stieltjes transform difference equation

$\left(t+b_{1}+1\right)\left(t+b_{2}+1\right)(t+1) S(t+1)-z\left(t+a_{1}\right)\left(t+a_{2}\right)\left(t+a_{3}\right) S(t)=\sum_{k=0}^{2} \xi_{k} t^{k}$

where

$$
\begin{aligned}
& \xi_{2}=(1-z) \nu_{0}, \quad \xi_{1}=\left[e_{1}(\mathbf{b}+1)-e_{1}(\mathbf{a}) z\right] \nu_{0}+(1-z) \nu_{1}, \\
& \xi_{0}=\left[e_{2}(\mathbf{b}+1)-e_{2}(\mathbf{a}) z\right] \nu_{0}+\left[e_{1}(\mathbf{b}+1)-e_{1}(\mathbf{a}) z-z\right] \nu_{1}+(1-z) \nu_{2},
\end{aligned}
$$

and $e_{1}, e_{2}, e_{3}$ denote the elementary symmetric polynomials defined by

$$
e_{1}(\mathbf{x})=\sum_{i} x_{i}, \quad e_{2}(\mathbf{x})=\sum_{i<j} x_{i} x_{j}, \quad e_{3}(\mathbf{x})=\sum_{i<j<k} x_{i} x_{j} x_{k} .
$$

\subsubsection{Uvarov Meixner polynomials}

Let $\omega \notin\{-a, 0\}$.

Special values

$$
a_{1} \rightarrow a, \quad a_{2} \rightarrow-\omega, \quad a_{3} \rightarrow-\omega+1, \quad b_{1} \rightarrow-\omega-1, \quad b_{2} \rightarrow-\omega .
$$

Linear functional

$$
L_{U}[r]=\sum_{x=0}^{\infty} r(x)(a)_{x} \frac{z^{x}}{x !}+M r(\omega) .
$$

Pearson equation

$$
\frac{\varrho_{U}(x+1)}{\varrho_{U}(x)}=\frac{z(x+a)(x-\omega)(x-\omega+1)}{(x-\omega)(x-\omega+1)(x+1)} .
$$


Moments

$$
\nu_{n}^{U}(z)=z^{n}(a)_{n}(1-z)^{-a-n}+M \phi_{n}(\omega), \quad n \in \mathbb{N}_{0} .
$$

Stieltjes transform difference equation

$$
\begin{aligned}
& (t-\omega)(t-\omega+1)\left[(t+1) S_{U}(t+1)-z(t+a) S_{U}(t)\right] \\
& =\left[(1-z) t^{2}+(-a z+2 \omega z-2 \omega-z+1) t+\omega(z+\omega-z \omega+2 a z-1)-a z\right] \nu_{0}^{U \varsigma} \\
& +[(1-z) t-2 z+2 z \omega-2 \omega-a z+1] \nu_{1}^{U}+(1-z) \nu_{2}^{U} .
\end{aligned}
$$

Stieltjes transform $M$-dependent difference equation

$$
(t+1) S_{U}(t+1)-z(t+a) S_{U}(t)=(1-z) \nu_{0}+M\left[\frac{t+1}{t+1-\omega}-\frac{z(t+a)}{t-\omega}\right] .
$$

\subsubsection{Symmetrized generalized Meixner polynomials}

Special values

$$
\begin{aligned}
& a_{1} \rightarrow a, \quad a_{2} \rightarrow-b-2 m, \quad a_{3} \rightarrow-2 m, \\
& b_{1} \rightarrow-a-2 m, \quad b_{2} \rightarrow b-2 m, \quad z \rightarrow-1, \quad x \rightarrow x+m .
\end{aligned}
$$

Linear functional

$$
L_{\varsigma}[r]=\varrho_{\varsigma}(0) \sum_{x=-m}^{m} r(x) \frac{(a+m)_{x}(-b-m)_{x}(-m)_{x}}{(-a-m+1)_{x}(b+1-m)_{x}} \frac{(-1)^{x}}{(m+1)_{x}},
$$

where

$$
\varrho_{\varsigma}(0)=\left(\begin{array}{c}
2 m \\
m
\end{array}\right) \frac{(a)_{m}(b+1+m)_{m}}{(a+m)_{m}(b+1-2 m)_{m}} .
$$

Pearson equation

$$
\frac{\varrho_{\varsigma}(x+1)}{\varrho_{\varsigma}(x)}=\frac{-(x+a+m)(x-b-m)(x-m)}{(x-a-m+1)(x+b+1+m)(x+1+m)} .
$$

Moments on the basis $\left\{\bar{\phi}_{n}(x)\right\}_{n \geq 0}$

$\nu_{n}^{\varsigma}=\frac{(-1)^{n}(a)_{n}(-b-2 m)_{n}(-2 m)_{n}}{(-a-2 m+1)_{n}(b-2 m+1)_{n}} F_{2}\left[\begin{array}{c}a+n,-b-2 m+n,-2 m+n \\ -a-2 m+1+n, b-2 m+1+n\end{array} ;-1\right], \quad n \in \mathbb{N}_{0}$.

Stieltjes transform difference equation

$$
\begin{aligned}
& (t-a-m+1)(t+b+1+m)(t+1+m) S_{\varsigma}(t+1)+(t+a+m)(t-b-m)(t-m) S_{\varsigma}(t) \\
& =\left[2 t^{2}+2(1-m) t+b-a-2 a b-4 a m+1\right] \nu_{0}^{\varsigma}+(2 t-4 m+3) \nu_{1}^{\varsigma}+2 \nu_{2}^{\varsigma} .
\end{aligned}
$$




\subsubsection{Reduced-Uvarov generalized Hahn polynomials of type I}

Let $\{\omega, \Omega\} \in\left\{\left(-a_{1}, a_{1}+1\right),\left(-a_{2}, a_{2}+1\right),(-b, b),(0,0)\right\}$.

Special values

$$
a_{3} \rightarrow \Omega, \quad b_{1} \rightarrow b, \quad b_{2} \rightarrow \Omega-1 .
$$

Linear functional

$$
L_{U}[r]=\sum_{x=0}^{\infty} r(x) \frac{\left(a_{1}\right)_{x}\left(a_{2}\right)_{x}}{(b+1)_{x}} \frac{z^{x}}{x !}+M r(\omega) .
$$

Pearson equation

$$
\frac{\varrho_{U}(x+1)}{\varrho_{U}(x)}=\frac{z\left(x+a_{1}\right)\left(x+a_{2}\right)(x+\Omega)}{(x+b+1)(x+\Omega)(x+1)} .
$$

Moments

$$
\nu_{n}^{U}(z)=z^{n} \frac{\left(a_{1}\right)_{n}\left(a_{2}\right)_{n}}{(b+1)_{n}}{ }_{2} F_{1}\left[\begin{array}{c}
a_{1}+n, a_{2}+n \\
b+1+n
\end{array} ; z\right]+M \phi_{n}(\omega), \quad n \in \mathbb{N}_{0} .
$$

Stieltjes transform difference equation

$$
\begin{aligned}
& (t+\Omega)\left[(t+b+1)(t+1) S_{U}(t+1)-z\left(t+a_{1}\right)\left(t+a_{2}\right) S_{U}(t)\right] \\
& =\left[(1-z) t^{2}+\left(b+\Omega-z \Omega-z a_{1}-z a_{2}+1\right) t-\left(\Omega a_{1}+\Omega a_{2}+a_{1} a_{2}\right) z+(b+1) \Omega\right] \nu_{0}^{U} \\
& +\left[(1-z) t+1-z\left(\Omega+a_{1}+a_{2}+1\right)+\Omega+b\right] \nu_{1}^{U}+(1-z) \nu_{2}^{U} .
\end{aligned}
$$

Stieltjes transform $M$-dependent difference equation

$$
\begin{aligned}
& (t+b+1)(t+1) S_{U}(t+1)-z\left(t+a_{1}\right)\left(t+a_{2}\right) S_{U}(t)=\left[t+b+1-z\left(t+a_{1}+a_{2}\right)\right] \nu_{0} \\
& +(1-z) \nu_{1}+M\left[\frac{(t+b+1)(t+1)}{t-\omega+1}-\frac{z\left(t+a_{1}\right)\left(t+a_{2}\right)}{t-\omega}\right] .
\end{aligned}
$$

\subsubsection{Christoffel generalized Hahn polynomials of type I}

Let $\omega \notin\left\{-a_{1},-a_{2},-b, 0, \frac{\nu_{1}}{\nu_{0}}\right\}$.

Special values

$$
a_{3} \rightarrow-\omega+1, \quad b_{1} \rightarrow b, \quad b_{2} \rightarrow-\omega-1 .
$$


Linear functional

$$
L_{C}[r]=\sum_{x=0}^{\infty} r(x)(x-\omega) \frac{\left(a_{1}\right)_{x}\left(a_{2}\right)_{x}}{(b+1)_{x}} \frac{z^{x}}{x !} .
$$

Pearson equation

$$
\frac{\varrho_{C}(x+1)}{\varrho_{C}(x)}=\frac{z\left(x+a_{1}\right)\left(x+a_{2}\right)(x+1-\omega)}{(x+b+1)(x+1)(x-\omega)}, \quad n \in \mathbb{N}_{0} .
$$

Moments

$$
\nu_{n}^{C}(z)=\nu_{n+1}+(n-\omega) \nu_{n}
$$

Stieltjes transform difference equation

$$
\begin{aligned}
& (t-\omega)(t+b+1)(t+1) S_{C}(t+1)-z(t-\omega+1)\left(t+a_{1}\right)\left(t+a_{2}\right) S_{C}(t) \\
& =\left[(z-1) \omega t^{2}-\left(\omega+b \omega-z \omega-\omega^{2}+z \omega^{2}-z \omega a_{1}-z \omega a_{2}-z a_{1} a_{2}\right) t\right] \nu_{0} \\
& +\left[\omega^{2}(b+1)-z(\omega-1)\left(\omega a_{1}+\omega a_{2}+a_{1} a_{2}\right)\right] \nu_{0}-(z-1)(t-\omega)(t-\omega+1) \nu_{1} .
\end{aligned}
$$

\subsubsection{Geronimus generalized Hahn polynomials of type I}

Let $\omega \notin\left\{-b-1,-1,1-a_{1}, 1-a_{2}\right\} \cup \mathbb{N}_{0}$.

Special values

$$
a_{3} \rightarrow-\omega, \quad b_{1} \rightarrow b, \quad b_{2} \rightarrow-\omega .
$$

Linear functional

$$
L_{G}[r]=\sum_{x=0}^{\infty} \frac{r(x)}{x-\omega} \frac{\left(a_{1}\right)_{x}\left(a_{2}\right)_{x}}{(b+1)_{x}} \frac{z^{x}}{x !}+M r(\omega) .
$$

Pearson equation

$$
\frac{\varrho_{G}(x+1)}{\varrho_{G}(x)}=\frac{z\left(x+a_{1}\right)\left(x+a_{2}\right)(x-\omega)}{(x+b+1)(x+1)(x+1-\omega)} .
$$

Moments

$$
\nu_{n}^{G}(z)=\phi_{n}(\omega)\left[M-S(\omega)+\sum_{k=0}^{n-1} \frac{\nu_{k}}{\phi_{k+1}(\omega)}\right], \quad n \in \mathbb{N}_{0} .
$$

Stieltjes transform difference equation

$$
\begin{aligned}
& (t-\omega+1)(t+b+1)(t+1) S_{G}(t+1)-z(t-\omega)\left(t+a_{1}\right)\left(t+a_{2}\right) S_{G}(t) \\
& =\left[(1-z) t+\left(b-z a_{1}-z a_{2}+1\right)\right] \nu_{0}+(1-z) \nu_{1} \\
& +\left[(t+b+1)(t+1)-z\left(t+a_{1}\right)\left(t+a_{2}\right)\right] \nu_{0}^{G} .
\end{aligned}
$$




\subsubsection{Truncated generalized Hahn polynomials of type I}

Special values

$$
a_{3} \rightarrow-N, \quad b_{1} \rightarrow b, \quad b_{2} \rightarrow-N-1 .
$$

Linear functional

$$
L_{T}[r]=\sum_{x=0}^{N} r(x) \frac{\left(a_{1}\right)_{x}\left(a_{2}\right)_{x}}{(b+1)_{x}} \frac{z^{x}}{x !}
$$

Pearson equation

$$
\frac{\varrho_{T}(x+1)}{\varrho_{T}(x)}=\frac{z\left(x+a_{1}\right)\left(x+a_{2}\right)(x-N)}{(x+b+1)(x-N)(x+1)} .
$$

Moments

$\nu_{n}^{T}(z)=\frac{\left(a_{1}\right)_{N}\left(a_{2}\right)_{N}}{(b+1)_{N}} \frac{z^{N}}{(N-n) !}{ }_{3} F_{2}\left[\begin{array}{c}n-N, 1,-N-b \\ 1-N-a_{1}, 1-N-a_{2}\end{array} ; \frac{1}{z}\right], \quad n \in \mathbb{N}_{0}$.

Stieltjes transform difference equation

$$
\begin{aligned}
& (t-N)\left[(t+b+1)(t+1) S_{U}(t+1)-z\left(t+a_{1}\right)\left(t+a_{2}\right) S_{U}(t)\right] \\
& =\left[(1-z) t^{2}+\left(b-N+z N-z a_{1}-z a_{2}+1\right) t-\left(-N a_{1}-N a_{2}+a_{1} a_{2}\right) z-(b+1) N\right] \nu_{0}^{U} \\
& +\left[(1-z) t+1-z\left(-N+a_{1}+a_{2}+1\right)-N+b\right] \nu_{1}^{U}+(1-z) \nu_{2}^{U} .
\end{aligned}
$$

\subsubsection{Symmetrized polynomials of type $(0,2)$}

Special values

$$
a_{1} \rightarrow-b_{1}-2 m, \quad a_{2} \rightarrow-b_{2}-2 m, \quad a_{3} \rightarrow-2 m, \quad z \rightarrow-1, \quad x \rightarrow x+m .
$$

Linear functional

$$
L_{\varsigma}[r]=\varrho_{\varsigma}(0) \sum_{x=-m}^{m} r(x) \frac{\left(-b_{1}-m\right)_{x}\left(-b_{2}-m\right)_{x}(-m)_{x}}{\left(b_{1}+1+m\right)_{x}\left(b_{2}+1+m\right)_{x}} \frac{(-1)^{x}}{(m+1)_{x}},
$$

where

$$
\varrho_{\varsigma}(0)=\left(\begin{array}{c}
2 m \\
m
\end{array}\right) \frac{\left(b_{1}+1+m\right)_{m}\left(b_{1}+1+m\right)_{m}}{\left(b_{1}+1\right)_{m}\left(b_{2}+1\right)_{m}}
$$


Pearson equation

$$
\frac{\varrho_{\varsigma}(x+1)}{\varrho_{\varsigma}(x)}=\frac{-\left(x-b_{1}-m\right)\left(x-b_{2}-m\right)(x-m)}{\left(x+b_{1}+1+m\right)\left(x+b_{2}+1+m\right)(x+1+m)} .
$$

Moments on the basis $\left\{\bar{\phi}_{n}(x)\right\}_{n \geq 0}$.

For $n \in \mathbb{N}_{0}$

$$
\nu_{n}^{\varsigma}=\frac{(-1)^{n}(-2 m)_{n}\left(-b_{1}-2 m\right)_{n}\left(-b_{2}-2 m\right)_{n}}{\left(b_{1}+1\right)_{n}\left(b_{2}+1\right)_{n}} F_{2}\left[\begin{array}{c}
-2 m+n,-b_{1}-2 m,-b_{2}-2 m+n, \\
b_{1}+1+n, b_{2}+1+n
\end{array} ; .\right.
$$

Stieltjes transform difference equation

$$
\begin{aligned}
& \left(t+b_{1}+1+m\right)\left(t+b_{2}+1+m\right)(t+1+m) S_{\varsigma}(t+1) \\
& +\left(t-b_{1}-m\right)\left(t-b_{2}-m\right)(t-m) S_{\varsigma}(t) \\
& =\left[2 t^{2}+2(1-m) t+8 m^{2}+2 m\left(2 b_{1}+2 b_{2}+1\right)+b_{1}+b_{2}+2 b_{1} b_{2}+1\right] \nu_{0}^{\varsigma} \\
& +(2 t-4 m+3) \nu_{1}^{\varsigma}+2 \nu_{2}^{\varsigma} .
\end{aligned}
$$

\subsection{Polynomials of type $(4,3 ; \mathrm{N}, 1)$}

Linear functional

$$
L[r]=\sum_{x=0}^{N} r(x) \frac{\left(a_{1}\right)_{x}\left(a_{2}\right)_{x}\left(a_{3}\right)_{x}(-N)_{x}}{\left(b_{1}+1\right)_{x}\left(b_{2}+1\right)_{x}\left(b_{3}+1\right)_{x}} \frac{1}{x !} .
$$

Pearson equation

$$
\frac{\varrho(x+1)}{\varrho(x)}=\frac{\left(x+a_{1}\right)\left(x+a_{2}\right)\left(x+a_{3}\right)(x-N)}{\left(x+b_{1}+1\right)\left(x+b_{2}+1\right)\left(x+b_{3}+1\right)(x+1)} .
$$

Moments

For $n \in \mathbb{N}_{0}$

$$
\nu_{n}=\frac{(-N)_{n}\left(a_{1}\right)_{n}\left(a_{2}\right)_{n}\left(a_{3}\right)_{n}}{\left(b_{1}+1\right)_{n}\left(b_{2}+1\right)_{n}\left(b_{3}+1\right)_{n}} F_{3}\left[\begin{array}{c}
-N+n, a_{1}+n, a_{2}+n, a_{3}+n \\
b_{1}+1+n, b_{2}+1+n, b_{3}+1+n
\end{array} ; 1\right] .
$$

Stieltjes transform difference equation

$$
\begin{aligned}
& \left(t+b_{1}+1\right)\left(t+b_{2}+1\right)\left(t+b_{3}+1\right)(t+1) S(t+1) \\
& -\left(t+a_{1}\right)\left(t+a_{2}\right)\left(t+a_{3}\right)(t-N) S(t)=\sum_{k=0}^{2} \xi_{k} t^{k}
\end{aligned}
$$


where

$\xi_{2}=\left[e_{1}(\mathbf{b}+1)-e_{1}(\mathbf{a})\right] \nu_{0}, \quad \xi_{1}=\left[e_{2}(\mathbf{b}+1)-e_{2}(\mathbf{a})\right] \nu_{0}+\left[e_{1}(\mathbf{b}+1)-e_{1}(\mathbf{a})-1\right] \nu_{1}$,

$\xi_{0}=\left[e_{3}(\mathbf{b}+1)-e_{3}(\mathbf{a})\right] \nu_{0}+\left[e_{2}(\mathbf{b}+1)-e_{2}(\mathbf{a})-e_{1}(\mathbf{a})-1\right] \nu_{1}+\left[e_{1}(\mathbf{b}+1)-e_{1}(\mathbf{a})-2\right] \nu_{2}$,

with $a_{4}=-N$.

If we use the relations

$e_{1}(\mathbf{a},-N)=e_{1}(\mathbf{a})-N, \quad e_{2}(\mathbf{a},-N)=e_{2}(\mathbf{a})-N e_{1}(\mathbf{a}), \quad e_{3}(\mathbf{a},-N)=e_{3}(\mathbf{a})-N e_{2}(\mathbf{a})$,

we obtain expressions for the coefficients $\xi_{k}$ explicitly depending on $N$

$$
\begin{aligned}
\xi_{2} & =\left[e_{1}(\mathbf{b}+1)-e_{1}(\mathbf{a})+N\right] \nu_{0}, \\
\xi_{1} & =\left[e_{2}(\mathbf{b}+1)-e_{2}(\mathbf{a})+N e_{1}(\mathbf{a})\right] \nu_{0}+\left[e_{1}(\mathbf{b}+1)-e_{1}(\mathbf{a})+N-1\right] \nu_{1}, \\
\xi_{0} & =\left[e_{3}(\mathbf{b}+1)-e_{3}(\mathbf{a})+N e_{2}(\mathbf{a})\right] \nu_{0}+\left[e_{2}(\mathbf{b}+1)-e_{2}(\mathbf{a})+(N-1)\left(e_{1}(\mathbf{a})+1\right)\right] \nu_{1} \\
& +\left[e_{1}(\mathbf{b}+1)-e_{1}(\mathbf{a})+N-2\right] \nu_{2} .
\end{aligned}
$$

\subsubsection{Uvarov Hahn polynomials}

Let $\omega \notin\{-a,-b, 0, N\}$.

Special values

$$
\begin{aligned}
& a_{1} \rightarrow a, \quad a_{2} \rightarrow-\omega, \quad a_{3} \rightarrow-\omega+1, \\
& b_{1} \rightarrow b, \quad b_{2} \rightarrow-\omega-1, \quad b_{3} \rightarrow-\omega .
\end{aligned}
$$

Linear functional

$$
L_{U}[r]=\sum_{x=0}^{N} r(x) \frac{(a)_{x}(-N)_{x}}{(b+1)_{x}} \frac{1}{x !}+M r(\omega) .
$$

Pearson equation

$$
\frac{\varrho_{U}(x+1)}{\varrho_{U}(x)}=\frac{(x+a)(x-\omega)(x-\omega+1)(x-N)}{(x+b+1)(x-\omega)(x-\omega+1)(x+1)} .
$$

Moments

$$
\nu_{n}^{U}=\frac{(-N)_{n}(a)_{n}}{(b+1)_{n}} \frac{(b+1-a)_{N-n}}{(b+1+n)_{N-n}}+M \phi_{n}(\omega), \quad n \in \mathbb{N}_{0} .
$$


Stieltjes transform difference equation

$$
\begin{aligned}
& (t-\omega)(t-\omega+1)\left[(t+b+1)(t+1) S_{U}(t+1)-(t+a)(t-N) S_{U}(t)\right] \\
& =\left[(N-a+b+1) t^{2}+(N-a+b-2 \omega-2 N \omega+2 a \omega-2 b \omega+N a+1) t\right] \nu_{0}^{U \varsigma} \\
& +\left(a \omega-N \omega-\omega-b \omega+\omega^{2}+N \omega^{2}-a \omega^{2}+b \omega^{2}+N a-2 N a \omega\right) \nu_{0}^{U \varsigma} \\
& +[(N-a+b) t+2 N-2 a+b-2 N \omega+2 a \omega-2 b \omega+N a-1] \nu_{1}^{U}+(-a-1+b+N) \nu_{2}^{U} .
\end{aligned}
$$

Stieltjes transform $M$-dependent difference equation

$$
\begin{aligned}
& (t+b+1)(t+1) S_{U}(t+1)-(t+a)(t-N) S_{U}(t) \\
& =(b+1-a+N) \nu_{0}+M\left[\frac{(t+b+1)(t+1)}{t+1-\omega}-\frac{(t+a)(t-N)}{t-\omega}\right] .
\end{aligned}
$$

\subsubsection{Symmetrized generalized Hahn polynomials of type I}

Special values

$$
\begin{aligned}
& a_{3} \rightarrow-b-N, \quad b_{1} \rightarrow-a_{1}-N, \quad b_{2} \rightarrow-a_{2}-N \\
& b_{3} \rightarrow b, \quad N \rightarrow 2 m, \quad x \rightarrow x+m .
\end{aligned}
$$

Linear functional

$L_{\varsigma}[r]=\varrho_{\varsigma}(0) \sum_{x=-m}^{m} r(x) \frac{\left(a_{1}+m\right)_{x}\left(a_{2}+m\right)_{x}(-b-m)_{x}(-m)_{x}}{\left(-a_{1}-m+1\right)_{x}\left(-a_{2}-m+1\right)_{x}(b+1+m)_{x}} \frac{1}{(m+1)_{x}}$,

where

$$
\varrho_{\varsigma}(0)=\left(\begin{array}{c}
2 m \\
m
\end{array}\right) \frac{\left(a_{1}\right)_{m}\left(a_{2}\right)_{m}(b+1+m)_{m}}{\left(a_{1}+m\right)_{m}\left(a_{2}+m\right)_{m}(b+1)_{m}} .
$$

Pearson equation

$$
\frac{\varrho_{\varsigma}(x+1)}{\varrho_{\varsigma}(x)}=\frac{\left(x+a_{1}+m\right)\left(x+a_{2}+m\right)(x-b-m)(x-m)}{\left(x+1-a_{1}-m\right)\left(x+1-a_{2}-m\right)(x+1+b+m)(x+1+m)} .
$$

Moments on the basis $\left\{\bar{\phi}_{n}(x)\right\}_{n \geq 0}$.

For $n \in \mathbb{N}_{0}$ 


$$
\begin{aligned}
\nu_{n}^{\varsigma} & =\frac{\left(a_{1}\right)_{n}\left(a_{2}\right)_{n}(-b-2 m)_{n}(-2 m)_{n}}{\left(-a_{1}-2 m+1\right)_{n}\left(-a_{2}-2 m+1\right)_{n}(b+1)_{n}} \\
& \times{ }_{4} F_{3}\left[\begin{array}{c}
-2 m+n, a_{1}+n, a_{2}+n,-b-2 m+n \\
-a_{1}-2 m+1+n,-a_{2}-2 m+1+n, b+1+n
\end{array} ; 1\right] .
\end{aligned}
$$

Stieltjes transform difference equation

$$
\begin{aligned}
& \left(t+1-a_{1}-m\right)\left(t+1-a_{2}-m\right)(t+1+b+m)(t+1+m) S_{\varsigma}(t+1) \\
& -\left(t+a_{1}+m\right)\left(t+a_{2}+m\right)(t-b-m)(t-m) S_{\varsigma}(t) \\
& =\left[2\left(b-a_{1}-a_{2}+1\right)(t+1-m) t+t(t+1)\right] \nu_{0}^{\varsigma} \\
& +\left[b-m-2 b m-m^{2}+1-(b+1)\left(a_{1}+a_{2}\right)+(2 b+4 m+1) a_{1} a_{2}\right] \nu_{0}^{\varsigma} \\
& +\left[(2 t+3-4 m)\left(b-a_{1}-a_{2}\right)+2(t+1-m)\right] \nu_{1}^{\varsigma}+\left(2 b+1-2 a_{1}-2 a_{2}\right) \nu_{2}^{\varsigma} .
\end{aligned}
$$

\subsubsection{Reduced-Uvarov generalized Hahn polynomials of type II}

Let $\{\omega, \Omega\} \in\left\{\left(-a_{1}, a_{1}+1\right),\left(-a_{2}, a_{2}+1\right),\left(-b_{1}, b_{1}\right),\left(-b_{2}, b_{2}\right),(0,0),(N,-N+1)\right\}$.

Special values

$$
a_{3} \rightarrow \Omega, \quad b_{3} \rightarrow \Omega-1
$$

Linear functional

$$
L_{U}[r]=\sum_{x=0}^{N} r(x) \frac{(-N)_{x}\left(a_{1}\right)_{x}\left(a_{2}\right)_{x}}{\left(b_{1}+1\right)_{x}\left(b_{2}+1\right)_{x}} \frac{1}{x !}+M r(\omega) .
$$

Pearson equation

$$
\frac{\varrho_{U}(x+1)}{\varrho_{U}(x)}=\frac{\left(x+a_{1}\right)\left(x+a_{2}\right)(x+\Omega)(x-N)}{\left(x+b_{1}+1\right)\left(x+b_{2}+1\right)(x+\Omega)(x+1)} .
$$

Moments

$$
\nu_{n}^{U}=\frac{(-N)_{n}\left(a_{1}\right)_{n}\left(a_{2}\right)_{n}}{\left(b_{1}+1\right)_{n}\left(b_{2}+1\right)_{n}}{ }_{3} F_{2}\left[\begin{array}{c}
-N+n, a_{1}+n, a_{2}+n \\
b_{1}+1+n, b_{2}+1+n
\end{array} ; 1\right]+M \phi_{n}(\omega), \quad n \in \mathbb{N}_{0} .
$$

Stieltjes transform difference equation

$$
\begin{aligned}
& (t+\Omega)\left[\left(t+b_{1}+1\right)\left(t+b_{2}+1\right)(t+1) S_{U}(t+1)-\left(t+a_{1}\right)\left(t+a_{2}\right)(t-N) S_{U}(t)\right] \\
& =\left[\left(N-a_{1}-a_{2}+b_{1}+b_{2}+2\right)(t+\Omega) t+\left(b_{1}+1\right)\left(b_{2}+1\right) t+N\left(a_{1}+a_{2}\right) t-a_{1} a_{2} t\right] \nu_{0}^{U} \\
& +\left[\left(b_{1}+1\right)\left(b_{2}+1\right) \Omega+a_{1} a_{2}(N-\Omega)+\left(a_{1}+a_{2}\right) \Omega N\right] \nu_{0}^{U} \\
& +\left[\left(N-a_{1}-a_{2}+b_{1}+b_{2}+1\right)(t+\Omega)+N+b_{1}+b_{2}+b_{1} b_{2}+(N-1)\left(a_{1}+a_{2}\right)-a_{1} a_{2}\right] \nu_{1}^{U} \\
& +\left(N+b_{1}+b_{2}-a_{1}-a_{2}\right) \nu_{2}^{U} .
\end{aligned}
$$


Stieltjes transform $M$-dependent difference equation

$$
\begin{aligned}
& \left(t+b_{1}+1\right)\left(t+b_{2}+1\right)(t+1) S_{U}(t+1)-\left(t+a_{1}\right)\left(t+a_{2}\right)(t-N) S_{U}(t) \\
& =\left[\left(N+2+b_{1}+b_{2}-a_{1}-a_{2}\right) t+b_{1}+b_{2}+b_{1} b_{2}+(N-1)\left(a_{1}+a_{2}\right)-a_{1} a_{2}+1\right] \nu_{0} \\
& +\left(N+b_{1}+b_{2}-a_{1}-a_{2}+1\right) \nu_{1} \\
& +M\left[\frac{\left(t+b_{1}+1\right)\left(t+b_{2}+1\right)(t+1)}{t-\omega+1}-\frac{\left(t+a_{1}\right)\left(t+a_{2}\right)(t-N)}{t-\omega}\right] .
\end{aligned}
$$

\subsubsection{Christoffel generalized Hahn polynomials of type II}

Let $\omega \notin\left\{-a_{1},-a_{2},-b_{1},-b_{2}, 0, N, \frac{\nu_{1}}{\nu_{0}}\right\}$.

Special values

$$
a_{3} \rightarrow-\omega+1, \quad b_{3} \rightarrow-\omega-1 .
$$

Linear functional

$$
L_{C}[r]=\sum_{x=0}^{\infty} r(x)(x-\omega) \frac{\left(a_{1}\right)_{x}\left(a_{2}\right)_{x}(-N)_{x}}{\left(b_{1}+1\right)_{x}\left(b_{2}+1\right)_{x}} \frac{1}{x !} .
$$

Pearson equation

$$
\frac{\varrho_{C}(x+1)}{\varrho_{C}(x)}=\frac{\left(x+a_{1}\right)\left(x+a_{2}\right)(x-N)(x+1-\omega)}{\left(x+b_{1}+1\right)\left(x+b_{2}+1\right)(x+1)(x-\omega)} .
$$

Moments

$$
\nu_{n}^{C}(z)=\nu_{n+1}+(n-\omega) \nu_{n}, \quad n \in \mathbb{N}_{0} .
$$

Stieltjes transform difference equation

$$
\begin{aligned}
& (t-\omega)\left(t+b_{1}+1\right)\left(t+b_{2}+1\right)(t+1) S_{C}(t+1)-(t-\omega+1)\left(t+a_{1}\right)\left(t+a_{2}\right)(t-N) S_{C}(t) \\
& =-\left[\omega\left(N+b_{1}+b_{2}+1\right)-(\omega-1)\left(a_{1}+a_{2}\right)\right] t^{2} \nu_{0}+\omega(\omega-1)\left(N+b_{1}+b_{2}+1\right) t \nu_{0} \\
& +\left[(-N+\omega) a_{1} a_{2}+\left(3 \omega-N \omega-\omega^{2}-1\right)\left(a_{1}+a_{2}\right)+\omega\left(\omega-b_{1} b_{2}\right)\right] t \nu_{0} \\
& +\left[\omega(\omega-1)(N-1)\left(a_{1}+a_{2}\right)+(\omega-1)(N-\omega) a_{1} a_{2}+\omega^{2}\left(b_{2}+1\right)\left(b_{1}+1\right)\right] \nu_{0} \\
& +\left(N-a_{1}-a_{2}+b_{1}+b_{2}+1\right)(t-\omega)(t-\omega+1) \nu_{1} .
\end{aligned}
$$




\subsubsection{Geronimus generalized Hahn polynomials of type II}

Let $\omega \notin\left\{-b_{1}-1,-b_{2}-1,-1,1-a_{1}, 1-a_{2}, 1+N\right\} \cup \mathbb{N}_{0}$.

Special values

$$
a_{3} \rightarrow-\omega, \quad b_{3} \rightarrow-\omega .
$$

Linear functional

$$
L_{G}[r]=\sum_{x=0}^{\infty} \frac{r(x)}{x-\omega} \frac{\left(a_{1}\right)_{x}\left(a_{2}\right)_{x}(-N)_{x}}{\left(b_{1}+1\right)_{x}\left(b_{2}+1\right)_{x}} \frac{1}{x !}+M r(\omega) .
$$

Pearson equation

$$
\frac{\varrho_{G}(x+1)}{\varrho_{G}(x)}=\frac{\left(x+a_{1}\right)\left(x+a_{2}\right)(x-N)(x-\omega)}{\left(x+b_{1}+1\right)\left(x+b_{2}+1\right)(x+1)(x+1-\omega)} .
$$

Moments

$$
\nu_{n}^{G}(z)=\phi_{n}(\omega)\left[M-S(\omega)+\sum_{k=0}^{n-1} \frac{\nu_{k}}{\phi_{k+1}(\omega)}\right], \quad n \in \mathbb{N}_{0} .
$$

Stieltjes transform difference equation

$$
\begin{aligned}
& (t-\omega+1)\left(t+b_{1}+1\right)\left(t+b_{2}+1\right)(t+1) S_{G}(t+1)-(t-\omega)\left(t+a_{1}\right)\left(t+a_{2}\right)(t-N) S_{G}(t) \\
& =\left[\left(N+2+b_{1}+b_{2}-a_{1}-a_{2}\right) t+\left(b_{1}+1\right)\left(b_{2}+1\right)+(N-1)\left(a_{1}+a_{2}\right)-a_{1} a_{2}\right] \nu_{0} \\
& +\left(N+b_{1}+b_{2}-a_{1}-a_{2}+1\right) \nu_{1} \\
& +\left[\left(N-a_{1}-a_{2}+b_{1}+b_{2}+3\right) t^{2}+\left(2 b_{1}+2 b_{2}+N a_{1}+N a_{2}-a_{1} a_{2}+b_{1} b_{2}+3\right) t\right] \nu_{0}^{G} \\
& +\left(b_{1}+b_{2}+b_{1} b_{2}+N a_{1} a_{2}+1\right) \nu_{0}^{G} .
\end{aligned}
$$

\subsubsection{Symmetrized polynomials of type $(1,2)$}

Special values

$a_{1} \rightarrow a, \quad a_{2} \rightarrow-b_{1}-N, \quad a_{3} \rightarrow-b_{2}-N, \quad b_{3} \rightarrow-a-N, \quad N \rightarrow 2 m, \quad x \rightarrow x+m$.

Linear functional

$$
L_{\varsigma}[r]=\varrho_{\varsigma}(0) \sum_{x=-m}^{m} r(x) \frac{(a+m)_{x}\left(-b_{1}-m\right)_{x}\left(-b_{2}-m\right)_{x}(-m)_{x}}{\left(b_{1}+1+m\right)_{x}\left(b_{2}+1+m\right)_{x}(-a-m+1)_{x}} \frac{1}{(m+1)_{x}},
$$


where

$$
\varrho_{\varsigma}(0)=\left(\begin{array}{c}
2 m \\
m
\end{array}\right) \frac{(a)_{m}\left(b_{1}+1+m\right)_{m}\left(b_{1}+1+m\right)_{m}}{(a+m)_{m}\left(b_{1}+1\right)_{m}\left(b_{2}+1\right)_{m}} .
$$

Pearson equation

$\frac{\varrho_{\varsigma}(x+1)}{\varrho_{\varsigma}(x)}=\frac{(x+a+m)\left(x-b_{1}-m\right)\left(x-b_{2}-m\right)(x-m)}{\left(x+b_{1}+1+m\right)\left(x+b_{2}+1+m\right)(x+1-a-m)(x+1+m)}$.

Moments on the basis $\left\{\bar{\phi}_{n}(x)\right\}_{n \geq 0}$

$$
\begin{aligned}
\nu_{n}^{\varsigma} & =\frac{(-2 m)_{n}(a)_{n}\left(-b_{1}-2 m\right)_{n}\left(-b_{2}-2 m\right)_{n}}{(1-a-2 m)_{n}\left(b_{1}+1\right)_{n}\left(b_{2}+1\right)_{n}} \\
& \times{ }_{4} F_{3}\left[\begin{array}{c}
-2 m+n, a+n,-b_{1}-2 m,-b_{2}-2 m+n, \\
1-a-2 m+n, b_{1}+1+n, b_{2}+1+n
\end{array} ; 1\right], \quad n \in \mathbb{N}_{0} .
\end{aligned}
$$

Stieltjes transform difference equation

$$
\begin{gathered}
(t+1-a-m)\left(t+b_{1}+1+m\right)\left(t+b_{2}+1+m\right)(t+1+m) S_{\varsigma}(t+1) \\
-(t+a+m)\left(t-b_{1}-m\right)\left(t-b_{2}-m\right)(t-m) S_{\varsigma}(t) \\
=\left[\left(4 m+3+2 b_{1}+2 b_{2}-2 a\right) t^{2}+2(m-1)\left(a-b_{1}-b_{2}\right) t\right] \nu_{0}^{\varsigma} \\
+\left[\left(-4 m^{2}+2 m+3\right) t-\left(18 a+4 a b_{1}+4 a b_{2}+1\right) m-\left(b_{1}+b_{2}+1\right)(a-1)-b_{1} b_{2}(2 a-1)\right] \nu_{0}^{\varsigma} \\
+\left[(2 t-4 m+3)\left(b_{1}+b_{2}-a\right)+2(t+2 m t+1)+4 m(1-2 m)\right] \nu_{1}^{\varsigma} \\
+\left(4 m+1+2 b_{1}+2 b_{2}-2 a\right) \nu_{2}^{\varsigma} .
\end{gathered}
$$

\section{Conclusions}

We have considered all solutions of the Pearson equation

$$
\frac{\varrho(x+1)}{\varrho(x)}=\frac{\eta(x)}{\sigma(x+1)},
$$

with $\operatorname{deg}(\sigma) \leq 4$ and $\operatorname{deg}(\sigma-\eta) \leq 3$. We deduce 15 canonical cases, from which 42 subcases can be obtained and we relate those to rational spectral transformations (Uvarov, Christoffel, and Geronimus), truncations, and symmetrizations. 
For each family, we have listed a representation of the moments and the first order linear difference equations satisfied by their Stieltjes transform.

Extensions to the class $s=3$ are possible, but the complexity of the formulas will demand new forms of notation in order to be able to type the results in a comprehensive way.

Acknowledgement 9 This work of the first author was done while visiting the Johannes Kepler Universität Linz and supported by the strategic program "Innovatives OÖ- 2010 plus" from the Upper Austrian Government. The second author acknowledges financial support of Dirección general de Investigación, Ministerio de Economía, Industria y Competitividad of Spain, grant MTM2015-65888. C4- 2-P.

\section{References}

[1] F. Abdelkarim and P. Maroni. The $D_{\omega}$-classical orthogonal polynomials. Results Math., 32(1-2):1-28, 1997.

[2] R. Álvarez Nodarse, J. Arvesú, and F. Marcellán. Modifications of quasidefinite linear functionals via addition of delta and derivatives of delta Dirac functions. Indag. Math. (N.S.), 15(1):1-20, 2004.

[3] R. Álvarez Nodarse, A. G. García, and F. Marcellán. On the properties for modifications of classical orthogonal polynomials of discrete variables. In Proceedings of the International Conference on Orthogonality, Moment Problems and Continued Fractions (Delft, 1994), volume 65, pages 3-18, 1995.

[4] R. Álvarez Nodarse and F. Marcellán. Difference equation for modifications of Meixner polynomials. J. Math. Anal. Appl., 194(1):250-258, 1995.

[5] R. Álvarez Nodarse and F. Marcellán. The modification of classical Hahn polynomials of a discrete variable. Integral Transform. Spec. Funct., 3(4):243-262, 1995.

[6] R. Álvarez Nodarse and J. Petronilho. On the Krall-type discrete polynomials. J. Math. Anal. Appl., 295(1):55-69, 2004. 
[7] I. Area, D. K. Dimitrov, E. Godoy, and V. Paschoa. Bounds for the zeros of symmetric Kravchuk polynomials. Numer. Algorithms, 69(3):611-624, 2015 .

[8] I. Area, E. Godoy, A. Ronveaux, and A. Zarzo. Classical symmetric orthogonal polynomials of a discrete variable. Integral Transforms Spec. Funct., 15(1):1-12, 2004.

[9] S. Belmehdi. On semi-classical linear functionals of class $s=1$. Classification and integral representations. Indag. Math. (N.S.), 3(3):253-275, 1992.

[10] L. Boelen, G. Filipuk, C. Smet, W. Van Assche, and L. Zhang. The generalized Krawtchouk polynomials and the fifth Painlevé equation. J. Difference Equ. Appl., 19(9):1437-1451, 2013.

[11] L. Boelen, G. Filipuk, and W. Van Assche. Recurrence coefficients of generalized Meixner polynomials and Painlevé equations. J. Phys. A, 44(3):035202, 19, 2011.

[12] M. I. Bueno and F. M. Dopico. A more accurate algorithm for computing the Christoffel transformation. J. Comput. Appl. Math., 205(1):567-582, 2007.

[13] M. I. Bueno and F. Marcellán. Darboux transformation and perturbation of linear functionals. Linear Algebra Appl., 384:215-242, 2004.

[14] E. B. Christoffel. Über die Gaußische Quadratur und eine Verallgemeinerung derselben. J. Reine Angew. Math., 55:61-82, 1858.

[15] M. Derevyagin, J. C. García-Ardila, and F. Marcellán. Multiple Geronimus transformations. Linear Algebra Appl., 454:158-183, 2014.

[16] M. Derevyagin and F. Marcellán. A note on the Geronimus transformation and Sobolev orthogonal polynomials. Numer. Algorithms, 67(2):271-287, 2014.

[17] D. Dominici. Laguerre-Freud equations for generalized Hahn polynomials of type I. J. Difference Equ. Appl., 24(6):916-940, 2018. 
[18] D. Dominici. Polynomial sequences associated with the moments of hypergeometric weights. SIGMA Symmetry Integrability Geom. Methods Appl., 12:Paper No. 044, 18, 2016.

[19] D. Dominici and F. Marcellán. Discrete semiclassical orthogonal polynomials of class one. Pacific J. Math., 268(2):389-411, 2014.

[20] S. Elaydi. An introduction to difference equations. Undergraduate Texts in Mathematics. Springer, New York, third edition, 2005.

[21] G. Filipuk and W. Van Assche. Discrete orthogonal polynomials with hypergeometric weights and Painlevé VI. SIGMA Symmetry Integrability Geom. Methods Appl., 14:Paper No. 088, 19, 2018.

[22] G. Filipuk and W. Van Assche. Recurrence coefficients of a new generalization of the Meixner polynomials. SIGMA Symmetry Integrability Geom. Methods Appl., 7:Paper 068, 11, 2011.

[23] D. Galant. Algebraic methods for modified orthogonal polynomials. Math. Comp., 59(200):541-546, 1992.

[24] A. G. García, F. Marcellán, and L. Salto. A distributional study of discrete classical orthogonal polynomials. In Proceedings of the Fourth International Symposium on Orthogonal Polynomials and their Applications (Evian-Les-Bains, 1992), volume 57, pages 147-162, 1995.

[25] W. Gautschi. On generating orthogonal polynomials. SIAM J. Sci. Statist. Comput., 3(3):289-317, 1982.

[26] J. Geronimus. On polynomials orthogonal with regard to a given sequence of numbers. Comm. Inst. Sci. Math. Méc. Univ. Kharkoff [Zapiski Inst. Mat. Mech.] (4), 17:3-18, 1940.

[27] J. Geronimus. Sur quelques propriétés des polynomes orthogonaux généralisés. Rec. Math. [Mat. Sbornik] N.S., 9 (51):121-135, 1941.

[28] E. Godoy, F. Marcellán, L. Salto, and A. Zarzo. Perturbations of discrete semiclassical functionals by Dirac masses. Integral Transform. Spec. Funct., 5(1-2):19-46, 1997. 
[29] M. N. Hounkonnou, C. Hounga, and A. Ronveaux. Discrete semiclassical orthogonal polynomials: generalized Charlier. J. Comput. Appl. Math., 114(2):361-366, 2000.

[30] M. E. H. Ismail. Classical and quantum orthogonal polynomials in one variable, volume 98 of Encyclopedia of Mathematics and its Applications. Cambridge University Press, Cambridge, 2005.

[31] R. Koekoek, P. A. Lesky, and R. F. Swarttouw. Hypergeometric orthogonal polynomials and their q-analogues. Springer Monographs in Mathematics. Springer-Verlag, Berlin, 2010.

[32] J. H. Lee and K. H. Kwon. Division problem of moment functionals. Rocky Mountain J. Math., 32(2):739-758, 2002. Conference on Special Functions (Tempe, AZ, 2000).

[33] F. Marcellán and P. Maroni. Sur l'adjonction d'une masse de Dirac à une forme régulière et semi-classique. Ann. Mat. Pura Appl. (4), 162:1-22, 1992.

[34] F. Marcellán and L. Salto. Discrete semi-classical orthogonal polynomials. J. Differ. Equations Appl., 4(5):463-496, 1998.

[35] F. Marcellán, M. Sghaier, and M. Zaatra. On semiclassical linear functionals of class $s=2$ : classification and integral representations. $J$. Difference Equ. Appl., 18(6):973-1000, 2012.

[36] P. Maroni. Une caractérisation des polynômes orthogonaux semiclassiques. C. R. Acad. Sci. Paris Sér. I Math., 301(6):269-272, 1985.

[37] P. Maroni. Prolégomènes à l'étude des polynômes orthogonaux semiclassiques. Ann. Mat. Pura Appl. (4), 149:165-184, 1987.

[38] P. Maroni. Sur la suite de polynômes orthogonaux associée à la forme $u=\delta_{c}+\lambda(x-c)^{-1}$ L. Period. Math. Hungar., 21(3):223-248, 1990.

[39] P. Maroni. Une théorie algébrique des polynômes orthogonaux. Application aux polynômes orthogonaux semi-classiques. In Orthogonal polynomials and their applications (Erice, 1990), volume 9 of IMACS Ann. Comput. Appl. Math., pages 95-130. Baltzer, Basel, 1991. 
[40] M. Masjed-Jamei and I. Area. A basic class of symmetric orthogonal polynomials of a discrete variable. J. Math. Anal. Appl., 399(1):291-305, 2013.

[41] M. Masjed-Jamei and I. Area. A symmetric generalization of SturmLiouville problems in discrete spaces. J. Difference Equ. Appl., 19(9):1544-1562, 2013.

[42] A. F. Nikiforov, S. K. Suslov, and V. B. Uvarov. Classical orthogonal polynomials of a discrete variable. Springer Series in Computational Physics. Springer-Verlag, Berlin, 1991.

[43] K. Oldham, J. Myland, and J. Spanier. An atlas of functions. Springer, New York, second edition, 2009.

[44] F. W. J. Olver, D. W. Lozier, R. F. Boisvert, and C. W. Clark, editors. NIST handbook of mathematical functions. U.S. Department of Commerce National Institute of Standards and Technology, Washington, DC, 2010 .

[45] F. Peherstorfer. Finite perturbations of orthogonal polynomials. $J$. Comput. Appl. Math., 44(3):275-302, 1992.

[46] A. Ronveaux and L. Salto. Discrete orthogonal polynomialspolynomial modification of a classical functional. J. Differ. Equations Appl., 7(3):323-344, 2001.

[47] M. Sghaier and M. Zaatra. A family of discrete semi-classical orthogonal polynomials of class one. Period. Math. Hungar., 76(1):68-87, 2018.

[48] V. B. Uvarov. The connection between systems of polynomials that are orthogonal with respect to different distribution functions. Ž. Vyčisl. Mat. i Mat. Fiz., 9:1253-1262, 1969.

[49] W. Van Assche. Orthogonal polynomials and Painlevé equations, volume 27 of Australian Mathematical Society Lecture Series. Cambridge University Press, Cambridge, 2018.

[50] A. Zhedanov. Rational spectral transformations and orthogonal polynomials. J. Comput. Appl. Math., 85(1):67-86, 1997. 